\title{
Cauchy Problem for Fractional Diffusion Equations
}

\author{
Samuil D. Eidelman and Anatoly N. Kochubei* \\ Institute of Mathematics, \\ National Academy of Sciences of Ukraine, \\ Tereshchenkivska 3, Kiev, 01601 Ukraine
}

Running head: "Fractional Diffusion Equations"

The address for correspondence:

\begin{abstract}
Anatoly N. Kochubei
Institute of Mathematics, National Academy of Sciences of Ukraine, Tereshchenkivska 3, Kiev, 01601 Ukraine.

E-mail: kochubei@i.com.ua
\end{abstract}

*Partially supported by CRDF under Grant UM1-2421-KV-02 


\begin{abstract}
We consider an evolution equation with the regularized fractional derivative of an order $\alpha \in(0,1)$ with respect to the time variable, and a uniformly elliptic operator with variable coefficients acting in the spatial variables. Such equations describe diffusion on inhomogeneous fractals. A fundamental solution of the Cauchy problem is constructed and investigated.
\end{abstract}

Key words: fractional diffusion equation; fractional derivative; Fox's H-function; fundamental solution; Levi method 


\section{INTRODUCTION}

In this paper we consider equations of the form

$$
\left(\mathbb{D}_{t}^{(\alpha)} u\right)(t, x)-B u(t, x)=f(t, x), \quad t \in(0, T], x \in \mathbb{R}^{n},
$$

where $\mathbb{D}_{t}^{(\alpha)}, 0<\alpha<1$, is a regularized fractional derivative (the Caputo derivative), that is

$$
\begin{aligned}
\left(\mathbb{D}_{t}^{(\alpha)} u\right)(t, x) & =\frac{1}{\Gamma(1-\alpha)}\left[\frac{\partial}{\partial t} \int_{0}^{t}(t-\tau)^{-\alpha} u(\tau, x) d \tau-t^{-\alpha} u(0, x)\right] \\
B & =\sum_{i, j=1}^{n} a_{i j}(x) \frac{\partial^{2}}{\partial x_{i} \partial x_{j}}+\sum_{j=1}^{n} b_{j}(x) \frac{\partial}{\partial x_{j}}+c(x)
\end{aligned}
$$

is a uniformly elliptic second order differential operator with bounded continuous real-valued coefficients.

A strong motivation for investigating such equations comes from physics. Fractional diffusion equations describe anomalous diffusion on fractals (physical objects of fractional dimension, like some amorphous semiconductors or strongly porous materials; see [1, 18 and references therein). In normal diffusion (described by the heat equation or more general parabolic equations) the mean square displacement of a diffusive particle behaves like const $t$ for $t \rightarrow \infty$. A typical behavior for anomalous diffusion is const $\cdot t^{\alpha}$, and this was the reason to invoke the equation (1.1), usually with $B=\Delta$, where this anomalous behavior is an easy mathematical fact. For connections to statistical mechanics see also [11, 17].

It is natural from the physical point of view to consider a usual Cauchy problem, with the initial condition

$$
u(0, x)=u_{0}(x)
$$

This setting determines the necessity to use the regularized fractional derivative (1.2). If, for example, one considers instead of (1.2) the Riemann-Liouville fractional derivative defined similarly, but without subtracting $t^{-\alpha} u(0, x)$, then the appropriate initial data will be the limit value, as $t \rightarrow 0$, of the fractional integral of a solution of the order $1-\alpha$, not the limit value of the solution itself (see e.g. [21, 15]).

Note that on a smooth enough function $v(t)$ the regularized fractional derivative $\mathbb{D}_{t}^{(\alpha)} v$ can

be written as $\left(\mathbb{D}^{(\alpha)} v\right)(t)=\frac{1}{\Gamma(1-\alpha)} \int_{0}^{t} \frac{v^{\prime}(t)}{(t-\tau)^{\alpha}} d \tau$. In the physical literature the expression on the right is used as the basic object for formulating fractional diffusion equations. However, in order to proceed rigorously, one has either to use (1.2), or to consider, as in [22], an equivalent integral equation instead of the Cauchy problem (1.1), (1.3). On the other hand, the above expression for $\mathbb{D}^{(\alpha)}$ on smooth functions is a special case of a generalized fractional differential operator of Dzhrbashyan-Nersessyan [6] who studied ordinary differential equations with such operators. For example, they showed that the solution of the Cauchy problem $\mathbb{D}^{(\alpha)} v-\lambda v=0$, $v(0)=1$, has the form $v(t)=E_{\alpha}\left(\lambda t^{\alpha}\right)$ where $E_{\alpha}$ is the Mittag-Leffler function. See also [19].

The mathematical theory of fractional diffusion equations has made only its first steps. An expression for the fundamental solution of the Cauchy problem (1.1), (1.3) with $B=\Delta$ was 
found independently by Schneider and Wyss [22] and Kochubei [13. It was also shown in [22] that the fundamental solution is non-negative, which led later [23, 24, 14, 27] to a probabilistic interpretation of the equation (1.1). In [22] only initial functions $u_{0} \in \mathcal{S}\left(\mathbb{R}^{n}\right)$ were considered. A more general situation was studied in [13] where $u_{0}$ was permitted to be unbounded, with minimal smoothness assumptions. There are also some results regarding initial-boundary value problems (see [26, 22]).

For general problems (1.1), (1.3), in [13] a uniqueness theorem for bounded solutions, and an exact uniqueness theorem (for the case $n=1$ ) for solutions with a possible exponential growth were proved; see Sect. 2 below. There are also several papers devoted to the Cauchy problem for abstract evolution equations (1.1), in which $B$ is a closed operator on a Banach space ([12, 8, 3, 4, 2, and others).

An obvious analogy with the classical theory of parabolic partial differential equations [7. 10. 16 suggests a wide range of problems for the general equations (1.1) which deserve to be investigated. It is natural to begin with the construction and investigation of a fundamental solution of the Cauchy problem (1.1), (1.3). That is the aim of this paper.

More specifically, we will construct and study, under natural assumptions upon the coefficients of $B$, a Green matrix for the problem (1.1), (1.3), that is such a pair

$$
\{Z(t, x ; \xi), Y(t, x ; \xi)\}, \quad\left(t \in(0, T], x, \xi \in \mathbb{R}^{n}\right)
$$

that for any bounded continuous function $u_{0}$ (locally Hölder continuous, if $n>1$ ) and any bounded function $f$, jointly continuous in $(t, x)$ and locally Hölder continuous in $x$, there exists a classical solution of the problem (1.1), (1.3) of the form

$$
u(t, x)=\int_{\mathbb{R}^{n}} Z(t, x ; \xi) u_{0}(\xi) d \xi+\int_{0}^{t} d \lambda \int_{\mathbb{R}^{n}} Y(t-\lambda, x, ; y) f(\lambda, y) d y .
$$

As in [13], we call a function $u(t, x)$ a classical solution if:

(i) $u(t, x)$ is twice continuously differentiable in $x$ for each $t>0$;

(ii) for each $x \in \mathbb{R}^{n} u(t, x)$ is continuous in $t$ on $[0, T]$, and its fractional integral

$$
\left(I_{0+}^{1-\alpha} u\right)(t, x)=\frac{1}{\Gamma(1-\alpha)} \int_{0}^{t}(t-\tau)^{-\alpha} u(\tau, x) d \tau
$$

is continuously differentiable in $t$ for $t>0$.

(iii) $u(t, x)$ satisfies (1.1) and (1.3).

The results of this paper (as well as those of the earlier one [13]) demonstrate a number of interesting features of the equation (1.1), which represent a peculiar union of properties typical for second order parabolic differential equations (like a kind of the maximum principle; see the proofs of Theorems 1,2 in [13]) and general parabolic equations and systems. It is well known [7, 10, 16] that the latter are characterized by the parabolic weight $2 b(b \geq 1)$, so that the differentiation in $t$ has the same "force" as the differentiation of the order $2 b$ with 
respect to the spatial variables. In other words, the differentiation in spatial variables has the weight $1 / 2 b$ with respect to $\partial / \partial t$. For the equation (1.1) we have $\alpha / 2$ instead of $1 / 2 b$, and this formal analogy goes through all the constructions and estimates including the description of uniqueness and correctness classes.

In contrast to classical parabolic equations, the fundamental solution $Z$ of the Cauchy problem (1.1), (1.3) (even for $B=\Delta$ ) has, if $n>1$, a singularity not only at $t=0$, but also at the "diagonal" $x=\xi$ with respect to the spatial variables. This causes serious complications for the implementation of the classical Levi method [7, 10, 16]. The estimates of the iterated kernels require a series of additional regularization procedures absent in the classical case. Moreover, while the classical heat kernel has a simple expression in elementary functions, in the fractional case we have to deal with Fox's H-functions and their asymptotics, which makes even simple (in principle) transformations and estimates difficult technical tasks. Note also that, in order to consider an inhomogeneous equation (1.1), we have to develop Levi's method twice - separately for $Z$ and $Y$.

The results can be generalized easily to the situation, in which all the coefficients of $B$ except the leading ones depend also on $t$. Moreover, if we consider only the case of the zero initial functions, that is we look only for the function $Y$, then we can include, without any changes, the general case of time-dependent coefficients. The same is true for the case $n=1$ which resembles the classical theory of parabolic equations. In fact, it seems probable that the stationarity assumption can be dropped completely. However, then the machinery of Fox's H-functions would not be available, and we would have to deal directly with contour integral representations of solutions of fractional diffusion equations with coefficients depending only on $t$. Their study is a complicated task in itself, and it looks reasonable to consider first the stationary case, in order to identify the main differences between the equations (1.1) and classical parabolic equations.

The main results of this paper are collected in Sect. 2. Sect. 3 contains the information regarding H-functions, and miscellaneous lemmas used subsequently. Proofs of parametrix estimates are given in Sect. 4 and used in Sect. 5 for substantiating the Levi method for the case where $n \geq 2$. The case $n=1$ is considered in Sect. 6. In Sect. 7 we prove the nonnegativity of the functions $Z$ and $Y$.

\section{MAIN RESULTS}

In this section we describe the assumptions on the coefficients and formulate principal results. The proofs will be given in subsequent sections.

2.1. Equations with constant coefficients. Let us begin with the case where the coefficients of $B$ are constant, and only the leading terms are present, so that

$$
B=\sum_{i, j=1}^{n} a_{i j} \frac{\partial^{2}}{\partial x_{i} \partial x_{j}} .
$$

We assume that the matrix $A=\left(a_{i j}\right)$ is positive definite.

The function $Z=Z_{0}(t, x-\xi)$ for this case is obtained by a change of variables from the fundamental solution found in [22, 13] for $B=\Delta$. In order to formulate the result, we recall the definition of Fox's H-function which will be used systematically in this paper. 
Let $\mu, \nu, p, q$ be integers satisfying the conditions $0 \leq \nu \leq p, 1 \leq \mu \leq q$. Suppose we have also the complex parameters $c_{1}, \ldots, c_{p}$ and $d_{1}, \ldots, d_{q}$, and positive real parameters $\gamma_{1}, \ldots, \gamma_{p}$ and $\delta_{1}, \ldots, \delta_{q}$, such that $P_{1} \cap P_{2}=\varnothing$ where

$$
\begin{gathered}
P_{1}=\left\{s=-\left(d_{j}+k\right) / \delta_{j}, j=1, \ldots, \mu ; k=0,1,2, \ldots\right\}, \\
P_{2}=\left\{s=\left(1-c_{j}+k\right) / \gamma_{j}, j=1, \ldots, \nu ; k=0,1,2, \ldots\right\} .
\end{gathered}
$$

We will need only the case where

$$
\rho \stackrel{\text { def }}{=} \sum_{k=1}^{q} \delta_{k}-\sum_{k=1}^{p} \gamma_{k}>0
$$

The H-function

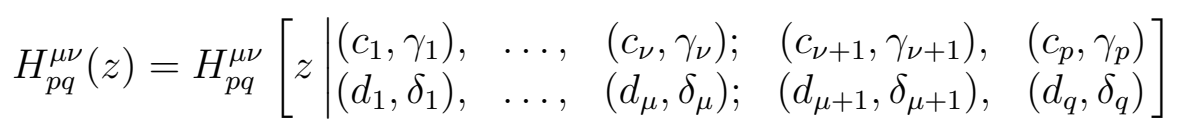

is defined by the contour integral

$$
H_{p q}^{\mu \nu}(z)=\frac{1}{2 \pi i} \int_{L} \frac{C(s) D(s)}{E(s) F(s)} z^{-s} d s
$$

with

$$
\begin{gathered}
C(s)=\prod_{k=1}^{\mu} \Gamma\left(d_{k}+\delta_{k} s\right), \quad D(s)=\prod_{k=1}^{\nu} \Gamma\left(1-c_{k}-\gamma_{k} s\right), \\
E(s)=\prod_{k=\mu+1}^{q} \Gamma\left(1-d_{k}-\delta_{k} s\right), \quad F(s)=\prod_{k=\nu+1}^{p} \Gamma\left(c_{k}+\gamma_{k} s\right)
\end{gathered}
$$

( $P_{1}$ and $P_{2}$ are the sets of poles for $C(s)$ and $D(s)$ respectively). The integration contour $L$ is an infinite loop running between $s=-\infty-i \sigma$ and $s=-\infty+i \sigma$ where $\sigma>\max _{1 \leq j \leq \mu}\left\{\left|\operatorname{Im} d_{j}\right| / \delta_{j}\right\}$ in such a way that $P_{1}$ lies to the left of $L$, and $P_{2}$ to the right of $L$.

Nearly all classical special functions can be represented as $\mathrm{H}$-functions with appropriate parameters. The theory of the H-function including its analytic properties, asymptotics, various relations, is expounded in [5, 20, 25. Below we formulate all the results we need.

Under the above assumptions the function $H_{p q}^{\mu \nu}(z)$ is holomorphic in a certain sector containing the positive real half-axis.

Now we can write the formula for the fundamental solution $Z_{0}$ :

$$
\begin{aligned}
Z_{0}(t, x-\xi)=\frac{\pi^{-n / 2}}{(\operatorname{det} A)^{1 / 2}}\left[\sum_{i, j=1}^{n}\right. & \left.A^{(i j)}\left(x_{i}-\xi_{i}\right)\left(x_{j}-\xi_{j}\right)\right]^{-n / 2} \\
& \times H_{12}^{20}\left[\frac{1}{4} t^{-\alpha} \sum_{i, j=1}^{n} A^{(i j)}\left(x_{i}-\xi_{i}\right)\left(x_{j}-\xi_{j}\right) \mid \begin{array}{ll}
(1, \alpha) & \\
\left(\frac{n}{2}, 1\right), & (1,1)
\end{array}\right]
\end{aligned}
$$


where $A=\left(A^{(i j)}\right)$ is the matrix inverse to $\left(a_{i j}\right)$. Two different proofs of $(2.3)$ can be found (for $B=\Delta$ ) in [13] and 22] (where an equivalent formula is given). In 13] we used the Fourier transform with respect to the spatial variables; the resulting equation is solved using the Mittag-Leffler function, and then the inverse Fourier transform is performed on the basis of the appropriate formulas for H-functions. The authors of 22] used the Mellin transform in $t$, the explicit expression of the Green function for the Laplacian, the inverse Mellin transform, and integration formulas for H-functions.

The function $Y=Y_{0}(t-\lambda, x-y)$ appearing in the representation (1.4), for this case has the form

$$
\begin{aligned}
Y_{0}(t-\lambda, x-y) & =\frac{\pi^{-n / 2}}{(\operatorname{det} A)^{1 / 2}}\left[\sum_{i, j=1}^{n} A^{(i j)}\left(x_{i}-y_{i}\right)\left(x_{j}-y_{j}\right)\right]^{-n / 2} \\
& \times(t-\lambda)^{\alpha-1} H_{12}^{20}\left[\frac{1}{4}(t-\lambda)^{-\alpha} \sum_{i, j=1}^{n} A^{(i j)}\left(x_{i}-y_{i}\right)\left(x_{j}-y_{j}\right) \mid \begin{array}{l}
(\alpha, \alpha) \\
\left(\frac{n}{2}, 1\right), \quad(1,1)
\end{array}\right] .
\end{aligned}
$$

In fact, $Y_{0}(t, x)$ is the Riemann-Liouville derivative of $Z_{0}(t, x)$ in $t$, of the order $1-\alpha$ (for $x \neq 0$, $Z_{0}(t, x) \rightarrow 0$ as $t \rightarrow 0$, so that the Riemann-Liouville derivative coincides in the case with the regularized fractional derivative).

Estimates of the function $Z_{0}$ and $Y_{0}$, and of their derivatives, are given in the following propositions. Denote $R=t^{-\alpha}|x|^{2}$. Here and below the letters $C, \sigma$ will denote various positive constants.

Proposition 1 (see [13]). (i) If $R \geq 1$, then

$$
\begin{gathered}
\left|D_{x}^{m} Z_{0}(t, x)\right| \leq C t^{-\frac{\alpha(n+m)}{2}} \exp \left(-\sigma t^{-\frac{\alpha}{2-\alpha}}|x|^{\frac{2}{2-\alpha}}\right), \quad|m| \leq 3, \\
\left|\mathbb{D}_{t}^{(\alpha)} Z_{0}(t, x)\right| \leq C t^{-\frac{\alpha(n+2)}{2}} \exp \left(-\sigma t^{-\frac{\alpha}{2-\alpha}}|x|^{\frac{2}{2-\alpha}}\right)
\end{gathered}
$$

(ii) If $R \leq 1, x \neq 0$, then

$$
\left|D_{x}^{m} Z_{0}(t, x)\right| \leq C t^{-\alpha}|x|^{-n+2-|m|}, \quad|m| \leq 3,
$$

if $n \geq 3$, or $n=2, m \neq 0$;

$$
\left|Z_{0}(t, x)\right| \leq C t^{-\alpha}\left[\left|\log \left(t^{-\alpha}|x|^{2}\right)\right|+1\right],
$$

if $n=2$;

$$
\left|\frac{\partial^{m} Z_{0}(t, x)}{\partial x^{m}}\right| \leq C t^{-\frac{(m+1) \alpha}{2}},
$$

if $n=1$.

(iii) If $R \leq 1, x \neq 0$, then

$$
\left|\mathbb{D}_{t}^{(\alpha)} Z_{0}(t, x)\right| \leq \begin{cases}C t^{-2 \alpha}|x|^{-n+2}, & \text { if } n \geq 3 \\ C t^{-\alpha}\left[\left|\log \left(t^{-\alpha}|x|^{2}\right)\right|+1\right], & \text { if } n=2 \\ C t^{-3 \alpha / 2}, & \text { if } n=1\end{cases}
$$


Note that the orders of the singularities at $x=0$ in (2.7), (2.8), and (2.10) are precise (they are based on the asymptotic expansions of the H-functions).

The next proposition contains estimates of the function $Y_{0}$ and its derivatives.

Proposition 2. (i) If $R \geq 1$, then

$$
\left|D_{x}^{m} Y_{0}(t, x)\right| \leq C t^{-\frac{\alpha(n+m)}{2}-1+\alpha} \exp \left(-\sigma t^{-\frac{\alpha}{2-\alpha}}|x|^{\frac{2}{2-\alpha}}\right), \quad|m| \leq 3,
$$

(ii) If $R \leq 1, x \neq 0, n>4$, then

$$
\left|D_{x}^{m} Y_{0}(t, x)\right| \leq C t^{-\alpha-1}|x|^{-n+4-|m|}, \quad|m| \leq 3,
$$

(iii) If $R \leq 1, x \neq 0, n=4$, then

$$
\begin{gathered}
\left|Y_{0}(t, x)\right| \leq C t^{-\alpha-1}\left[\left|\log \left(t^{-\alpha}|x|^{2}\right)\right|+1\right] \\
\left|D_{x} Y_{0}(t, x)\right| \leq C t^{-\frac{3 \alpha}{2}-1}, \\
\left|D_{x}^{m} Y_{0}(t, x)\right| \leq C t^{-2 \alpha-1}\left[\left|\log \left(t^{-\alpha}|x|^{2}\right)\right|+1\right], \quad|m|=2, \\
\left|D_{x}^{m} Y_{0}(t, x)\right| \leq C t^{-2 \alpha-1}|x|^{-1}\left[\left|\log \left(t^{-\alpha}|x|^{2}\right)\right|+1\right], \quad|m|=3 .
\end{gathered}
$$

(iv) If $R \leq 1, x \neq 0, n=3$, then

$$
\begin{gathered}
\left|Y_{0}(t, x)\right| \leq C t^{-\frac{\alpha}{2}-1} \\
\left|D_{x} Y_{0}(t, x)\right| \leq C t^{-\alpha-1} \\
\left|D_{x}^{m} Y_{0}(t, x)\right| \leq C t^{-\alpha-1}|x|^{-1}, \quad|m|=2, \\
\left|D_{x}^{m} Y_{0}(t, x)\right| \leq C t^{-\alpha-1}|x|^{-2}, \quad|m|=3 .
\end{gathered}
$$

(v) If $R \leq 1, x \neq 0, n=2$, then

$$
\begin{gathered}
\left|Y_{0}(t, x)\right| \leq C t^{-1}, \\
\left|D_{x} Y_{0}(t, x)\right| \leq C t^{-\frac{\alpha}{2}-1}, \\
\left|D_{x}^{m} Y_{0}(t, x)\right| \leq C t^{-\alpha-1}\left[\left|\log \left(t^{-\alpha}|x|^{2}\right)\right|+1\right], \quad|m|=2, \\
\left|D_{x}^{m} Y_{0}(t, x)\right| \leq C t^{-\alpha-1}|x|^{-1}\left[\left|\log \left(t^{-\alpha}|x|^{2}\right)\right|+1\right], \quad|m|=3 .
\end{gathered}
$$


(vi) If $R \leq 1, x \neq 0, n=1$, then

$$
\left|D_{x}^{m} Y_{0}(t, x)\right| \leq C t^{-\frac{(m-1) \alpha}{2}-1}, \quad m=0,1,2,3 .
$$

2.2. The general case. We make the following assumptions on the coefficients of the operator $B$.

$\left(\mathbf{B}_{1}\right)$ The coefficients $a_{i j}(x), b_{j}(x), c(x)$ are bounded Hölder continuous functions on $\mathbb{R}^{n}$.

$\left(\mathbf{B}_{2}\right)$ The uniform parabolicity condition: there exists such a constant $\delta>0$ that for any $x, \xi \in \mathbb{R}^{n}$

$$
\sum_{i, j=1}^{n} a_{i j}(x) \xi_{i} \xi_{j} \geq \delta|\xi|^{2}
$$

In order to describe regularity properties of solutions of the equation (1.1) with respect to the variable $t$, it is convenient to introduce the class $H_{\mu}^{\alpha+\lambda}[0, T]$ of such functions $\varphi(t)$ that $t^{\mu} \varphi(t)$ is Hölder continuous on $[0, T]$ with the exponent $\alpha+\lambda, \lambda>0$. It was proved in [13] that the problem (1.1), (1.3) cannot have more than one bounded classical solution belonging (for each fixed $x \in \mathbb{R}^{n}$ ) to $H_{\mu}^{\alpha+\lambda}[0, T]$, if $0<\lambda<1-\alpha, 0 \leq \mu<\lambda+1$.

Below we denote by $\gamma$ various Hölder exponents with respect to spatial variables (without restricting generality they will be assumed equal).

Theorem. a) There exists a Green matrix $\{Z(t, x ; \xi), Y(t, x ; \xi)\}$ for the problem (1.1), (1.3), of the form

$$
\begin{aligned}
& Z(t, x ; \xi)=Z_{0}(t, x-\xi ; \xi)+V_{Z}(t, x ; \xi), \\
& Y(t, x ; \xi)=Y_{0}(t, x-\xi ; \xi)+V_{Y}(t, x ; \xi),
\end{aligned}
$$

where $\left(Z_{0}, Y_{0}\right)$, the Green matrix of the Cauchy problem for the equation obtained by "freezing" the coefficients $a_{i j}$ at the parameter point $\xi$ and setting other coefficients equal to zero, satisfies the estimates listed in Propositions 1,2, with the constants independent of $\xi \in \mathbb{R}^{n}$. The functions $V_{Z}, V_{Y}$ satisfy the estimates

(i) If $n=1$, then

$$
\begin{gathered}
\left|D_{x}^{m} V_{Z}(t, x ; \xi)\right| \leq C t^{(\gamma-m-1) \alpha / 2} \exp \left(-\sigma t^{-\frac{\alpha}{2-\alpha}}|x-\xi|^{\frac{2}{2-\alpha}}\right), \\
\left|D_{x}^{m} V_{Y}(t, x ; \xi)\right| \leq C t^{\alpha-1+(\gamma-m-1) \alpha / 2} \exp \left(-\sigma t^{-\frac{\alpha}{2-\alpha}}|x-\xi|^{\frac{2}{2-\alpha}}\right),
\end{gathered}
$$

$m=0,1,2$.

(ii) If $n=2$, then

$$
\begin{gathered}
\left|V_{Z}(t, x ; \xi)\right| \leq C t^{\frac{\gamma \alpha}{2}-\alpha} \exp \left(-\sigma t^{-\frac{\alpha}{2-\alpha}}|x-\xi|^{\frac{2}{2-\alpha}}\right), \\
\left|D_{x} V_{Z}(t, x ; \xi)\right| \leq C t^{\frac{\gamma_{0} \alpha}{2}-\alpha}|x-\xi|^{-1+\gamma-\gamma_{0}} \exp \left(-\sigma t^{-\frac{\alpha}{2-\alpha}}|x-\xi|^{\frac{2}{2-\alpha}}\right), \\
\left|V_{Y}(t, x ; \xi)\right| \leq C t^{\frac{\gamma \alpha}{2}-1} \exp \left(-\sigma t^{-\frac{\alpha}{2-\alpha}}|x-\xi|^{\frac{2}{2-\alpha}}\right), \\
\left|D_{x} V_{Y}(t, x ; \xi)\right| \leq C t^{\frac{\gamma_{0} \alpha}{2}-1}|x-\xi|^{-1+\gamma-\gamma_{0}} \exp \left(-\sigma t^{-\frac{\alpha}{2-\alpha}}|x-\xi|^{\frac{2}{2-\alpha}}\right),
\end{gathered}
$$


where $\gamma_{0}<\gamma$ is an arbitrary fixed positive constant (here and below in the formulas containing $\gamma_{0}$ the constant $C$ depends on $\gamma_{0}$ ).

(iii) If $n=3$, then

$$
\begin{gathered}
\left|D_{x}^{m} V_{Z}(t, x ; \xi)\right| \leq C t^{\frac{\gamma_{0} \alpha}{2}-\alpha}|x-\xi|^{-1-|m|+\gamma-\gamma_{0}} \exp \left(-\sigma t^{-\frac{\alpha}{2-\alpha}}|x-\xi|^{\frac{2}{2-\alpha}}\right), \\
\left|D_{x}^{m} V_{Y}(t, x ; \xi)\right| \leq C t^{\left(\gamma_{0}+\gamma\right) \frac{\alpha}{4}-1}|x-\xi|^{-1-|m|+\left(\gamma-\gamma_{0}\right) / 2} \exp \left(-\sigma t^{-\frac{\alpha}{2-\alpha}}|x-\xi|^{\frac{2}{2-\alpha}}\right)
\end{gathered}
$$

$|m|=0,1$.

(iv) If $n=4$, then

$$
\begin{aligned}
& \left|D_{x}^{m} V_{Z}(t, x ; \xi)\right| \leq C t^{\frac{\left(\gamma-\gamma_{0}\right) \alpha}{2}-\alpha}|x-\xi|^{-2-|m|+\gamma-2 \gamma_{0}} \exp \left(-\sigma t^{-\frac{\alpha}{2-\alpha}}|x-\xi|^{\frac{2}{2-\alpha}}\right), \\
& \left|D_{x}^{m} V_{Y}(t, x ; \xi)\right| \leq C t^{\frac{\left(\gamma-\gamma_{0}\right) \alpha}{4}-1}|x-\xi|^{-2-|m|+\gamma-2 \gamma_{0}} \exp \left(-\sigma t^{-\frac{\alpha}{2-\alpha}}|x-\xi|^{\frac{2}{2-\alpha}}\right), \\
|m|=0,1 ; \gamma_{0}<\gamma / 2 . &
\end{aligned}
$$

(v) If $n \geq 5$, then

$$
\begin{aligned}
& \left|D_{x}^{m} V_{Z}(t, x ; \xi)\right| \leq C t^{\frac{\gamma_{0} \alpha}{2}-\alpha}|x-\xi|^{2-n-|m|+\gamma-\gamma_{0}} \exp \left(-\sigma t^{-\frac{\alpha}{2-\alpha}}|x-\xi|^{\frac{2}{2-\alpha}}\right), \\
& \left|D_{x}^{m} V_{Y}(t, x ; \xi)\right| \leq C t^{\left(\gamma_{0}+\gamma\right) \frac{\alpha}{4}-1}|x-\xi|^{2-n-|m|+\left(\gamma-\gamma_{0}\right) / 2} \exp \left(-\sigma t^{-\frac{\alpha}{2-\alpha}}|x-\xi|^{\frac{2}{2-\alpha}}\right), \\
& |m|=0,1 .
\end{aligned}
$$

(vi) If $n \geq 2,|m|=2$, then

$$
\begin{aligned}
& \left|D_{x}^{m} V_{Z}(t, x ; \xi)\right| \leq C t^{\gamma_{1}-\alpha}|x-\xi|^{-n+\gamma_{2}} \exp \left(-\sigma t^{-\frac{\alpha}{2-\alpha}}|x-\xi|^{\frac{2}{2-\alpha}}\right) \\
& \left|D_{x}^{m} V_{Y}(t, x ; \xi)\right| \leq C t^{\gamma_{1}-1}|x-\xi|^{-n+\gamma_{2}} \exp \left(-\sigma t^{-\frac{\alpha}{2-\alpha}}|x-\xi|^{\frac{2}{2-\alpha}}\right)
\end{aligned}
$$

where $\gamma_{1}, \gamma_{2}$ are some positive constants.

(vii) The estimates for the fractional derivatives $\mathbb{D}_{t}^{(\alpha)} V_{Z}, \mathbb{D}_{t}^{(\alpha)} V_{Y}$ of the functions $Z, Y$ are the same as the above estimates for their second order derivatives.

b) Let $u(t, x)$ be the solution (1.4) of the problem (1.1), (1.3) with a bounded continuous function $u_{0}$ (locally Hölder continuous if $n>1$ ) and a bounded jointly continuous and locally Hölder continuous, in $x$, function $f$. If $0<\lambda<1-\alpha, \alpha+\lambda<\nu$, the for any $x \in \mathbb{R}^{n}$ $u(\cdot, x) \in H_{\nu}^{\alpha+\lambda}[0, T]$.

c) The functions $Z$ and $Y$ are nonnegative.

The rest of this paper is devoted to the proof of the Theorem. As it was mentioned in the Introduction, it can be generalized to the nonstationary case, and some lemmas below are formulated in a way general enough to cover that more general situation. 


\section{AUXILIARY RESULTS}

3.1. H-functions. We collect here some results regarding $\mathrm{H}$-functions, which will be used below. For the proofs and further details see [5, 20, 25].

a) Differentiation formulas.

$$
\begin{aligned}
& \frac{d}{d z} H_{12}^{20}\left[z \mid \begin{array}{ll}
\left(c_{1}, \gamma_{1}\right) \\
\left(d_{1}, \delta_{1}\right), & \left(d_{2}, \delta_{2}\right)
\end{array}\right]=-z^{-1} H_{23}^{30}\left[z \mid \begin{array}{lll}
\left(c_{1}, \gamma_{1}\right), & (0,1) \\
\left(d_{1}, \delta_{1}\right), & \left(d_{2}, \delta_{2}\right), & (1,1)
\end{array}\right] . \\
& \frac{d}{d z} H_{23}^{30}\left[z \mid \begin{array}{lll}
\left(c_{1}, \gamma_{1}\right), & \left(c_{2}, \gamma_{2}\right) \\
\left(d_{1}, \delta_{1}\right), & \left(d_{2}, \delta_{2}\right), & \left(d_{3}, \delta_{3}\right)
\end{array}\right]=-z^{-1} H_{34}^{40}\left[z \mid \begin{array}{llll}
\left(c_{1}, \gamma_{1}\right), & \left(c_{2}, \gamma_{2}\right), & (0,1) & \\
\left(d_{1}, \delta_{1}\right), & \left(d_{2}, \delta_{2}\right), & \left(d_{3}, \gamma_{3}\right) & (1,1)
\end{array}\right] .
\end{aligned}
$$

In a similar way $\frac{d}{d z} H_{34}^{40}$ is expressed via $H_{45}^{50}$ etc.

By $(2.2)$,

$$
H_{12}^{20}\left[z \mid \begin{array}{l}
(1, \alpha) \\
\left(\frac{n}{2}, 1\right), \quad(1,1)
\end{array}\right]=\frac{1}{2 \pi i} \int_{L} \frac{\Gamma\left(\frac{n}{2}+s\right) \Gamma(1+s)}{\Gamma(1+\alpha s)} z^{-s} d s .
$$

We will need this function with $z=\omega(t-\tau)^{-\alpha}, \omega>0, t>\tau$. We have

$$
u(t)=H_{12}^{20}\left[\omega(t-\tau)^{-\alpha} \mid \begin{array}{l}
(1, \alpha) \\
\left(\frac{n}{2}, 1\right), \quad(1,1)
\end{array}\right]=\frac{1}{2 \pi i} \int_{L} \frac{\Gamma\left(\frac{n}{2}+s\right) \Gamma(1+s)}{\Gamma(1+\alpha s)} \omega^{-s}(t-\tau)^{\alpha s} d s
$$

In order to calculate the Riemann-Liouville fractional derivative $\left(D_{\tau+}^{\alpha} u\right)(t)=\frac{1}{\Gamma(1-\alpha)} \frac{\partial}{\partial t} \int_{\tau}^{t}(t-$ $s)^{-\alpha} u(s) d s$, we note that $D_{\tau+}^{\alpha}$ transforms $(t-\tau)^{\alpha s}$ into $\frac{\Gamma(1+\alpha s)}{\Gamma(1-\alpha+\alpha s)}(t-\tau)^{\alpha s-\alpha}$ (see [21]). Now we find from (3.4) that

$$
\left(D_{\tau+}^{\alpha} u\right)(t)=(t-\tau)^{-\alpha} H_{12}^{20}\left[\omega(t-\tau)^{-\alpha} \mid \begin{array}{cc}
(1-\alpha, \alpha) & \\
\left(\frac{n}{2}, 1\right), & (1,1)
\end{array}\right]
$$

Similarly

$$
\left(D_{\tau+}^{1-\alpha} u\right)(t)=(t-\tau)^{\alpha-1} H_{12}^{20}\left[\omega(t-\tau)^{-\alpha} \mid \begin{array}{ll}
(\alpha, \alpha) & \\
\left(\frac{n}{2}, 1\right), & (1,1)
\end{array}\right]
$$

b) Asymptotics at infinity. The asymptotic behavior of $\mathrm{H}$-functions for $z \rightarrow \infty$ has been thoroughly investigated. We will need only one result of this kind, for a specific class of $\mathrm{H}-$ functions, for a real argument, with only the leading term of the asymptotic expansion:

$$
H_{p \mu}^{\mu 0}\left[z \mid \begin{array}{lll}
\left(c_{1}, \gamma_{1}\right), & \ldots, & \left(c_{p}, \gamma_{p}\right) \\
\left(d_{1}, \delta_{1}\right), & \ldots, & \left(d_{\mu}, \delta_{\mu}\right)
\end{array}\right] \sim \text { const } \cdot z^{(1-a) / \rho} \exp \left(-b^{1 / \rho} \rho z^{1 / \rho}\right)
$$

where $a=\sum_{k=1}^{p} c_{k}-\sum_{k=1}^{\mu} d_{k}+\frac{1}{2}(\mu-p+1), b=\prod_{k=1}^{p} \gamma_{k}^{\gamma_{k}} \prod_{l=1}^{\mu} \delta_{l}^{-\delta_{l}}$, and $\rho$ is given by $(2.1)$. 
In particular, from (3.7) we get estimates of some specific H-functions for $|z| \geq 1$ :

$$
\begin{aligned}
& \left|H_{12}^{20}\left[z \mid \begin{array}{l}
(1, \alpha) \\
\left(\frac{n}{2}, 1\right),
\end{array} \quad(1,1)\right]\right| \leq C|z|^{\frac{n}{2(2-\alpha)}} \exp \left(-\sigma|z|^{\frac{1}{2-\alpha}}\right) ;
\end{aligned}
$$

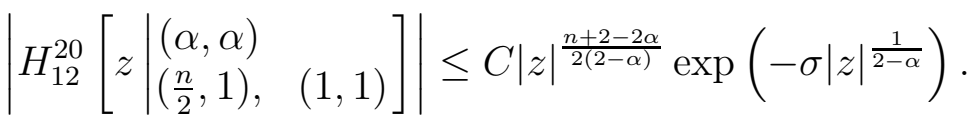

Similar estimates hold for functions appearing in expressions for derivatives of the above $\mathrm{H}-$ functions. In all cases the estimates contain the same exponentially decreasing factor while the degrees of positive powers of $|z|$ are different and specific for each case.

c) Asymptotics near the origin. In order to obtain, for the above class of H-functions, an asymptotic expansion near the origin, we write an $\mathrm{H}$-function as

$$
H_{p \mu}^{\mu 0}\left[z \mid \begin{array}{lll}
\left(c_{1}, \gamma_{1}\right), & \ldots, & \left(c_{p}, \gamma_{p}\right) \\
\left(d_{1}, \delta_{1}\right), & \ldots, & \left(d_{\mu}, \delta_{\mu}\right)
\end{array}\right]=\sum_{s \in P_{1}} \operatorname{Res}\left(\frac{C(s)}{F(s)} z^{-s}\right) .
$$

In specific cases, writing $P_{1}$ explicitly and using well-known properties of the function Gamma, we find a required number of terms in asymptotic expansions. In particular, for $z \rightarrow+0$

$$
H_{12}^{20}\left[z \mid \begin{array}{ll}
(1, \alpha) \\
\left(\frac{n}{2}, 1\right),
\end{array} \quad(1,1)\right]= \begin{cases}\frac{\Gamma\left(\frac{n}{2}-1\right)}{\Gamma(1-\alpha)} z+O\left(z^{\min \left(\frac{n}{2}, 2\right)}\right), & \text { if } n \geq 3 \\
-\frac{z \log z}{\Gamma(1-\alpha)}+O(z), & \text { if } n=2 \\
\frac{\sqrt{\pi}}{\Gamma\left(1-\frac{\alpha}{2}\right)} z^{1 / 2}-\frac{2 \sqrt{\pi}}{\Gamma(1-\alpha)} z+\frac{2 \sqrt{\pi}}{\Gamma\left(1-\frac{3 \alpha}{2}\right)} z^{3 / 2}+O\left(z^{2}\right), & \text { if } n=1\end{cases}
$$

(here and below we present those terms of the asymptotic expansions which are actually used in this work and in [13]),

$$
\begin{aligned}
& H_{23}^{30}\left[z \mid \begin{array}{ll}
(1, \alpha), & (0,1), \\
\left(\frac{n}{2}, 1\right), & (1,1), \quad(1,1)
\end{array}\right]= \begin{cases}-\frac{\Gamma\left(\frac{n}{2}-1\right)}{\Gamma(1-\alpha)} z+O\left(z^{\min \left(\frac{n}{2}, 2\right)}\right), & \text { if } n \geq 3, \\
\frac{z \log z}{\Gamma(1-\alpha)}+O(z), & \text { if } n=2, \\
-\frac{\sqrt{\pi}}{2 \Gamma\left(1-\frac{\alpha}{2}\right)} z^{1 / 2}+\frac{2 \sqrt{\pi}}{\Gamma(1-\alpha)} z-\frac{3 \sqrt{\pi}}{\Gamma\left(1-\frac{3 \alpha}{2}\right)} z^{3 / 2}+O\left(z^{2}\right), & \text { if } n=1\end{cases} \\
& H_{34}^{40}\left[z \mid \begin{array}{lll}
(1, \alpha), & (0,1), & (0,1) \\
\left(\frac{n}{2}, 1\right), & (1,1), & (1,1), \quad(1,1)
\end{array}\right]= \begin{cases}\frac{\Gamma\left(\frac{n}{2}-1\right)}{\Gamma(1-\alpha)} z+O\left(z^{\min \left(\frac{n}{2}, 2\right)}\right), & \text { if } n \geq 3 \\
-\frac{z \log z}{\Gamma(1-\alpha)}+O(z), & \text { if } n=2, \\
\frac{\sqrt{\pi}}{4 \Gamma\left(1-\frac{\alpha}{2}\right)} z^{1 / 2}-\frac{2 \sqrt{\pi}}{\Gamma(1-\alpha)} z & \\
+\frac{9 \sqrt{\pi}}{2 \Gamma\left(1-\frac{3 \alpha}{2}\right)} z^{3 / 2}+O\left(z^{2}\right), & \text { if } n=1\end{cases}
\end{aligned}
$$

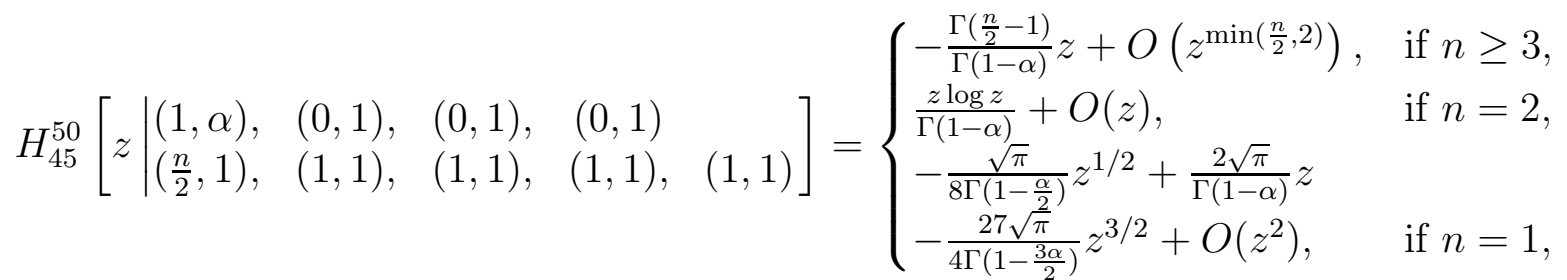


Next we give the expansions for the function appearing in (2.4) and those emerging in the course of differentiating (2.4). A different character of their asymptotics is caused by the fact that in this case the pole $s=1$ appears both in the numerator and denominator of the fraction in (2.2) and cancel each other. We have

$$
H_{12}^{20}\left[z \mid \begin{array}{ll}
(\alpha, \alpha) \\
\left(\frac{n}{2}, 1\right), \quad(1,1)
\end{array}\right]= \begin{cases}-\frac{\Gamma\left(\frac{n}{2}-2\right)}{\Gamma(-\alpha)} z^{2}+O\left(z^{\min \left(\frac{n}{2}, 3\right)}\right), & \text { if } n \geq 5, \\
\frac{z^{2} \log z}{\Gamma(-\alpha)}+\gamma_{1} z^{2}+O\left(z^{3} \log z\right), & \text { if } n=4, \\
-\frac{2 \sqrt{\pi}}{\Gamma\left(-\frac{\alpha}{2}\right)} z^{3 / 2}+\frac{2 \sqrt{\pi}}{\Gamma(-\alpha)} z^{2}+O\left(z^{5 / 2}\right), & \text { if } n=3, \\
\alpha z-\frac{z^{2} \log z}{\Gamma(-\alpha)}+\gamma_{2} z^{2}+O\left(z^{3} \log z\right), & \text { if } n=2, \\
\frac{\sqrt{\pi}}{\Gamma\left(\frac{\alpha}{2}\right)} z^{1 / 2}+\frac{2 \sqrt{\pi}}{\Gamma\left(-\frac{\alpha}{2}\right)} z^{3 / 2}+O\left(z^{2}\right), & \text { if } n=1,\end{cases}
$$

where $\gamma_{1}, \gamma_{2}$ are certain constants. Next,

$$
\begin{aligned}
& H_{23}^{30}\left[z \mid \begin{array}{ll}
(\alpha, \alpha), & (0,1) \\
\left(\frac{n}{2}, 1\right), & (1,1), \quad(1,1)
\end{array}\right]= \begin{cases}\frac{2 \Gamma\left(\frac{n}{2}-2\right)}{\Gamma(-\alpha)} z^{2}+O\left(z^{\min \left(\frac{n}{2}, 3\right)}\right), & \text { if } n \geq 5, \\
-2 \frac{z^{2} \log z}{\Gamma(-\alpha)}-2 \gamma_{1} z^{2}+O\left(z^{3} \log z\right), & \text { if } n=4, \\
\frac{3 \sqrt{\pi}}{\Gamma\left(-\frac{\alpha}{2}\right)} z^{3 / 2}-\frac{4 \sqrt{\pi}}{\Gamma(-\alpha)} z^{2}+O\left(z^{5 / 2}\right), & \text { if } n=3, \\
-\alpha z-2 \frac{z^{2} \log z}{\Gamma(-\alpha)}-2 \gamma_{2} z^{2}+O\left(z^{3} \log z\right), & \text { if } n=2, \\
-\frac{\sqrt{\pi}}{2 \Gamma\left(\frac{\alpha}{2}\right)} z^{1 / 2}-\frac{3 \sqrt{\pi}}{\Gamma\left(-\frac{\alpha}{2}\right)} z^{3 / 2}+O\left(z^{2}\right), & \text { if } n=1,\end{cases}
\end{aligned}
$$

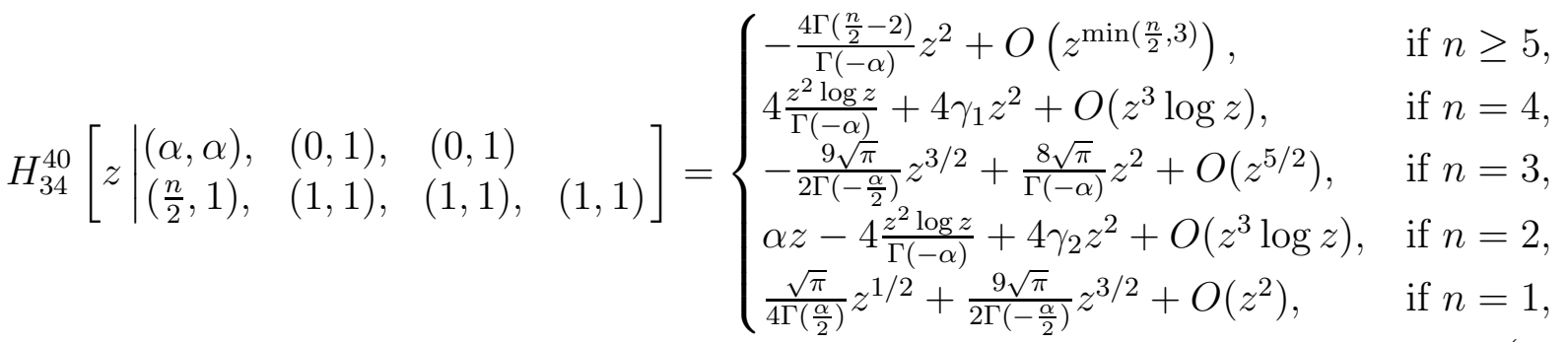

Finally,

$$
\begin{aligned}
& H_{45}^{50}\left[z \mid \begin{array}{lllll}
(\alpha, \alpha), & (0,1), & (0,1), & (0,1) & \\
\left(\frac{n}{2}, 1\right), & (1,1), & (1,1), & (1,1), & (1,1)
\end{array}\right] \\
& = \begin{cases}\frac{8 \Gamma\left(\frac{n}{2}-2\right)}{\Gamma(-\alpha)} z^{2}+O\left(z^{\min \left(\frac{n}{2}, 3\right)}\right), & \text { if } n \geq 5, \\
-8 \frac{z^{2} \log z}{\Gamma(-\alpha)}-8 \gamma_{1} z^{2}+O\left(z^{3} \log z\right), & \text { if } n=4, \\
\frac{27 \sqrt{\pi}}{4 \Gamma\left(-\frac{\alpha}{2}\right)} z^{3 / 2}-\frac{16 \sqrt{\pi}}{\Gamma(-\alpha)} z^{2}+O\left(z^{5 / 2}\right), & \text { if } n=3, \\
-\alpha z+8 \frac{z^{2} \log z}{\Gamma(-\alpha)}-8 \gamma_{2} z^{2}+O\left(z^{3} \log z\right), & \text { if } n=2, \\
-\frac{\sqrt{\pi}}{8 \Gamma\left(\frac{\alpha}{2}\right)} z^{1 / 2}-\frac{27 \sqrt{\pi}}{4 \Gamma\left(-\frac{\alpha}{2}\right)} z^{3 / 2}+O\left(z^{2}\right), & \text { if } n=1,\end{cases}
\end{aligned}
$$

3.2. Miscellaneous lemmas. Let us fix $\beta \in(0,1)$. For any $x, y \in \mathbb{R}^{n}, \lambda<t$ denote

$$
\rho(t, x ; \lambda, y)=\left(\frac{|x-y|}{(t-\lambda)^{\beta}}\right)^{\frac{1}{1-\beta}} .
$$


Lemma 1. Suppose that $0 \leq \tau<\lambda<t \leq T, x, y, \xi \in \mathbb{R}^{n}$. Then

$$
\rho(t, x ; \lambda, y)+\rho(\lambda, y ; \tau, \xi) \geq \rho(t, x ; \tau, \xi) .
$$

Proof. Let $f(y)=\rho(t, x ; \lambda, y)+\rho(\lambda, y ; \tau, \xi)$. Introducing a new variable $\widehat{y}=y-x$ we can write

$$
f(y)=f_{1}(\widehat{y})=\left(\frac{|\widehat{y}|}{(t-\lambda)^{\beta}}\right)^{\frac{1}{1-\beta}}+\left(\frac{|\widehat{y}-(\xi-x)|}{(\lambda-\tau)^{\beta}}\right)^{\frac{1}{1-\beta}} .
$$

Denoting

$$
f_{2}(r)=\left(\frac{r}{(t-\lambda)^{\beta}}\right)^{\frac{1}{1-\beta}}+\left(\frac{|r-| \xi-x||}{(\lambda-\tau)^{\beta}}\right)^{\frac{1}{1-\beta}}, \quad r \geq 0
$$

we find that

$$
\min _{y \in \mathbb{R}^{n}} f(y)=\min _{y \in \mathbb{R}^{n}} f_{1}(y) \geq \min _{r \geq 0} f_{2}(r) .
$$

The derivative $f_{2}^{\prime}(r)$ is positive for $r>|\xi-x|$; thus $f_{2}(r)$ attains its minimum value on the interval $[0,|x-\xi|]$. A standard search for its possible local extremum points yields a single point, in which the function takes the value $\left(\frac{|\xi-x|}{(t-\tau)^{\beta}}\right)^{\frac{1}{1-\beta}}$. On the other hand,

$$
f_{2}(0)=\left(\frac{|\xi-x|}{(\lambda-\tau)^{\beta}}\right)^{\frac{1}{1-\beta}}, \quad f_{2}(|x-\xi|)=\left(\frac{|\xi-x|}{(t-\lambda)^{\beta}}\right)^{\frac{1}{1-\beta}}
$$

whence $f_{2}(r) \geq\left(\frac{|\xi-x|}{(t-\tau)^{\beta}}\right)^{\frac{1}{1-\beta}}$. Together with (3.19) this implies (3.18).

The next three lemmas establish integral inequalities involving the function $\rho$. We keep the notation of Lemma 1.

Lemma 2. For any $\mu>0, \varepsilon \in(0,1)$

$$
\begin{aligned}
& {[(t-\lambda)(\lambda-\tau)]^{-\beta n} \int_{\mathbb{R}^{n}} \exp \{-\mu[\rho(t, x ; \lambda, y)}+\rho(\lambda, y ; \tau, \xi)]\} \\
& \leq C(\varepsilon)(t-\tau)^{-n \beta} \exp \{-(1-\varepsilon) \mu \rho(t, x ; \tau, \xi)\} .
\end{aligned}
$$

This lemma is a consequence of Lemma 1. See the proof of Lemma 5.1 in [7] where a similar result was obtained for $n=1$.

Lemma 3. For any $a_{1}, a_{2}>0$, any $b_{1}, b_{2}>0$, such that $b_{1}+b_{2}<n$, and any $\mu>0, \varepsilon \in(0,1)$

$$
\begin{gathered}
\int_{\tau}^{t}(t-\lambda)^{a_{1}-1}(\lambda-\tau)^{a_{2}-1} \int_{\mathbb{R}^{n}}|x-y|^{b_{1}-n}|y-\xi|^{b_{2}-n} \exp \{-\mu[\rho(t, x ; \lambda, y)+\rho(\lambda, y ; \tau, \xi)]\} d y d \lambda \\
\leq C\left(\varepsilon, a_{1}, a_{2}, b_{1}, b_{2}\right)(t-\tau)^{a_{1}+a_{2}-1}|x-\xi|^{b_{1}+b_{2}-n} \exp \{-(1-\varepsilon) \mu \rho(t, x ; \tau, \xi)\} .
\end{gathered}
$$


Proof. Writing $\mathbb{R}^{n}=\bigcup_{k=1}^{4} V_{k}$ where

$$
\begin{aligned}
& V_{1}=\left\{y \in \mathbb{R}^{n}:|x-y|>(t-\lambda)^{\beta},|y-\xi|>(\lambda-\tau)^{\beta}\right\}, \\
& V_{2}=\left\{y \in \mathbb{R}^{n}:|x-y|>(t-\lambda)^{\beta},|y-\xi| \leq(\lambda-\tau)^{\beta}\right\}, \\
& V_{3}=\left\{y \in \mathbb{R}^{n}:|x-y| \leq(t-\lambda)^{\beta},|y-\xi|>(\lambda-\tau)^{\beta}\right\}, \\
& V_{1}=\left\{y \in \mathbb{R}^{n}:|x-y| \leq(t-\lambda)^{\beta},|y-\xi| \leq(\lambda-\tau)^{\beta}\right\},
\end{aligned}
$$

we decompose the left-hand side in (3.21) into the sum of four integrals $J_{1}, J_{2}, J_{3}, J_{4}$.

If $y \in V_{1}$, then $|x-y|^{b_{1}-n}<(t-\lambda)^{\beta\left(b_{1}-n\right)},|y-\xi|^{b_{2}-n}<(\lambda-\tau)^{\beta\left(b_{2}-n\right)}$, so that

$$
\begin{aligned}
J_{1} \leq \int_{\tau}^{t}(t-\lambda)^{a_{1}+\beta b_{1}-1}(\lambda-\tau)^{a_{2}+\beta b_{2}-1}[(t-\lambda) & (\lambda-\tau)]^{-n \beta} \\
& \times \int_{\mathbb{R}^{n}} \exp \{-\mu[\rho(t, x ; \lambda, y)+\rho(\lambda, y ; \tau, \xi)]\} d y d \lambda,
\end{aligned}
$$

and by Lemma 2

$$
J_{1} \leq C(t-\tau)^{a_{1}+a_{2}+\left(b_{1}+b_{2}-n\right) \beta-1} \exp \left\{-\left(1-\frac{\varepsilon}{2}\right) \mu \rho(t, x ; \tau, \xi)\right\} .
$$

Since

$$
\begin{aligned}
& (t-\tau)^{\left(b_{1}+b_{2}-n\right) \beta} \exp \left\{-\frac{\varepsilon}{2} \mu \rho(t, x ; \tau, \xi)\right\} \\
& \quad=[\rho(t, x ; \tau, \xi)]^{(1-\beta)\left(n-b_{1}-b_{2}\right)} \exp \left\{-\frac{\varepsilon}{2} \mu \rho(t, x ; \tau, \xi)\right\}|x-\xi|^{b_{1}+b_{2}-n} \leq C|x-\xi|^{b_{1}+b_{2}-n}
\end{aligned}
$$

the inequality (3.22) means that $J_{1}$ does not exceed the right-hand side of (3.21).

In order to estimate $J_{2}$, we consider two distinct cases. Suppose first that $|x-\xi| \geq 2(t-\tau)^{\beta}$. Then $|x-y| \geq(t-\tau)^{\beta}$, so that by Lemma 1

$$
\begin{gathered}
J_{2} \leq(t-\tau)^{\beta\left(b_{1}-n\right)} \exp \{-\mu \rho(t, x ; \tau, \xi)\} \int_{\tau}^{t}(t-\lambda)^{a_{1}-1}(\lambda-\tau)^{a_{2}-1} d \lambda \int_{|y-\xi| \leq(\lambda-\tau)^{\beta}}|y-\xi|^{b_{2}-n} d y \\
=C(t-\tau)^{a_{1}+a_{2}-1}(t-\tau)^{\beta\left(b_{1}+b_{2}-n\right)} \exp \{-\mu \rho(t, x ; \tau, \xi)\} \\
\quad \leq C(\varepsilon)(t-\tau)^{a_{1}+a_{2}-1}|x-\xi|^{b_{1}+b_{2}-n} \exp \{-(1-\varepsilon) \mu \rho(t, x ; \tau, \xi)\} .
\end{gathered}
$$

If $|x-\xi|<2(t-\tau)^{\beta}$, then $|x-y|<3(t-\tau)^{\beta}$,

$$
J_{2} \leq \exp \{-\mu \rho(t, x ; \tau, \xi)\} \int_{\tau}^{t}(t-\lambda)^{a_{1}-1}(\lambda-\tau)^{a_{2}-1} d \lambda \int_{|y-\xi|<3(t-\tau)^{\beta}}|x-y|^{b_{1}-n}|y-\xi|^{b_{2}-n} d y
$$

and by Lemma 2 from Chapter 1 of [10] we get

$$
J_{2} \leq C(t-\tau)^{a_{1}+a_{2}-1}|x-\xi|^{b_{1}+b_{2}-n} \exp \{-\mu \rho(t, x ; \tau, \xi)\}
$$


Together with (3.23) this yields the required bound for $J_{2}$.

The estimate for $J_{3}$ is proved similarly to that of $J_{2}$.

Let us consider $J_{4}$. It follows from the definition of $V_{4}$ that $J_{4}=0$ if $|x-\xi| \geq 2(t-\tau)^{\beta}$. Therefore we may assume that $|x-\xi|<2(t-\tau)^{\beta}$ and note that

$$
V_{4} \subset\left\{y \in \mathbb{R}^{n}:|y-\xi|<2(t-\tau)^{\beta}\right\} .
$$

Then

$$
J_{4} \leq \exp \{-\mu \rho(t, x ; \tau, \xi)\} \int_{\tau}^{t}(t-\lambda)^{a_{1}-1}(\lambda-\tau)^{a_{2}-1} d \lambda \int_{|y-\xi|<2(t-\tau)^{\beta}}|x-y|^{b_{1}-n}|y-\xi|^{b_{2}-n} d y .
$$

Using again Lemma 2 from Chapter 1 of [10] we find that

$$
J_{4} \leq C(t-\tau)^{a_{1}+a_{2}-1}|x-\xi|^{b_{1}+b_{2}-n} \exp \{-\mu \rho(t, x ; \tau, \xi)\},
$$

as desired.

Remark 1. If $b_{1}+b_{2}>n$, then the assertion of Lemma 3 still holds if the factor $|x-\xi|^{b_{1}+b_{2}-n}$ is omitted from the right-hand side of (3.21). The proof is similar; we have only to use the appropriate estimate from the same lemma of [10].

Lemma 4. If $0 \leq c_{2}<c_{1}<1, \gamma>0$, then

$$
\begin{aligned}
\int_{\mathbb{R}^{n}}|x-y|^{-n+\gamma} \exp \left\{-\mu\left[c_{1} \rho(t, x ; \lambda, y)+c_{2} \rho(\lambda, y ; \tau, \xi)\right]\right\} d y & \\
& \leq C \exp \left\{-\mu c_{2} \rho(t, x ; \tau, \xi)\right\}(t-\lambda)^{\beta \gamma}
\end{aligned}
$$

where the constant depends on $c_{2}-c_{1}$.

Proof. Let us write the integral in the left-hand side of (3.24) as the sum $I_{1}+I_{2}$ of two integrals corresponding to the decomposition $\mathbb{R}^{n}=W_{1} \cup W_{2}$ where $W_{1}=\left\{y \in \mathbb{R}^{n}:|y-x| \leq\right.$ $\left.(t-\lambda)^{\beta}\right\}, W_{2}=\left\{y \in \mathbb{R}^{n}:|y-x|>(t-\lambda)^{\beta}\right\}$.

Using Lemma 1, we get

$$
I_{1} \leq \exp \left\{-\mu c_{2} \rho(t, x ; \tau, \xi)\right\} \int_{|y-x| \leq(t-\lambda)^{\beta}}|x-y|^{-n+\gamma} d y=C(t-\lambda)^{\beta \gamma} \exp \left\{-\mu c_{2} \rho(t, x ; \tau, \xi)\right\} .
$$

Next, again by Lemma 1,

$$
\begin{array}{r}
I_{2}=\int_{|y-x|>(t-\lambda)^{\beta}}|x-y|^{-n+\gamma} \exp \left\{-\mu\left[c_{1} \rho(t, x ; \lambda, y)+c_{2} \rho(\lambda, y ; \tau, \xi)\right]\right\} d y \\
\leq(t-\lambda)^{-n \beta+\gamma \beta} \exp \left\{-\mu c_{2} \rho(t, x ; \tau, \xi)\right\} \int_{\mathbb{R}^{n}} \exp \left\{-\mu\left(c_{1}-c_{2}\right) \rho(t, x ; \lambda, y) d y\right\} \\
\leq(t-\lambda)^{\beta \gamma} \exp \left\{-\mu c_{2} \rho(t, x ; \tau, \xi)\right\},
\end{array}
$$


as desired.

The next two lemmas give estimates of the iterated kernels

$$
K_{m}(t, x ; \tau, \xi)=\int_{\tau}^{t} d \lambda \int_{\mathbb{R}^{n}} K_{1}(t, x ; \lambda, y) K_{m-1}(\lambda, y ; \tau, \xi) d y, \quad m \geq 2
$$

where $K_{1}=K$ is a given kernel. We will treat the cases $n \geq 2$ and $n=1$ separately.

Lemma 5. Let $n \geq 2$. Suppose that $K(t, x ; \tau, \xi)$ is a continuous function on

$$
\mathcal{P}_{n}=\left\{(t, x ; \tau, \xi): x, \xi \in \mathbb{R}^{n}, x \neq \xi, 0 \leq \tau<t \leq T\right\},
$$

such that

$$
|K(t, x ; \tau, \xi)| \leq A_{1}(t-\tau)^{\nu_{0} \beta-1}|x-\xi|^{-n+\nu_{1}} \exp \{-\mu \rho(t, x ; \tau, \xi)\}
$$

where $A_{1}, \mu>0,0<\nu_{0}, \nu_{1}, \beta<1$, and $n \nu_{1}^{-1}$ is not an integer. Then the series $\mathcal{R}(t, x ; \tau, \xi)=$ $\sum_{m=1}^{\infty} K_{m}(t, x ; \tau, \xi)$ is absolutely and uniformly convergent on $\mathcal{P}_{n} \cap\{(t, x ; \tau, \xi): t-\tau \geq \delta>$ $0,|x-\xi| \geq \delta>0\}$, and

$$
|\mathcal{R}(t, x ; \tau, \xi)| \leq C(t-\tau)^{\nu_{0} \beta-1}|x-\xi|^{-n+\nu_{1}} \exp \left\{-\mu^{*} \rho(t, x ; \tau, \xi)\right\},
$$

with some $\mu^{*} \in(0, \mu)$.

Proof. The bounds for the kernels (3.25) are obtained by two stages. First we use Lemma 3. We find, for a small $\varepsilon>0$, that

$$
\left|K_{2}(t, x ; \tau, \xi)\right| \leq C_{2}(\varepsilon)(t-\tau)^{2 \nu_{0} \beta-1}|x-\xi|^{-n+2 \nu_{1}} \exp \{-\mu(1-\varepsilon) \rho(t, x ; \tau, \xi)\},
$$

and so on, so that

$$
\left|K_{s}(t, x ; \tau, \xi)\right| \leq C_{s}(\varepsilon)(t-\tau)^{s \nu_{0} \beta-1}|x-\xi|^{-n+s \nu_{1}} \exp \{-\mu(1-(s-1) \varepsilon) \rho(t, x ; \tau, \xi)\},
$$

whenever $-n+s \nu_{1}<0$. Let $s^{*}=\min \left\{s:-n+s \nu_{1}>0\right\}$. Then by Remark 1

$$
\left|K_{s^{*}}(t, x ; \tau, \xi)\right| \leq C_{0}(t-\tau)^{a_{0}-1} \exp \left\{-\mu^{*} \rho(t, x ; \tau, \xi)\right\},
$$

where $a_{0}=s^{*} \nu_{0} \beta, \mu^{*}=\left[1-\left(s^{*}-1\right) \varepsilon\right] \mu$.

For all the next iterations, we use (3.26), (3.29), and Lemma 4. Using the identity

$$
\int_{\tau}^{t}(t-\lambda)^{a_{1}-1}(\lambda-\tau)^{a_{2}-1} d \lambda=B\left(a_{1}, a_{2}\right)(t-\tau)^{a_{1}+a_{2}-1}
$$

we get by induction that

$$
\begin{aligned}
\left|K_{s^{*}+m}(t, x ; \tau, \xi)\right| \leq C_{0} C_{1}^{m}\left\{\prod_{j=1}^{m} B\left(\left(\nu_{0}+\nu_{1}\right) \beta, a_{0}+\left(\nu_{0}+\nu_{1}\right)(j-1)\right)\right\} \\
\times(t-\tau)^{m\left(\nu_{0}+\nu_{1}\right) \beta+a_{0}} \exp \left\{-\mu^{*} \rho(t, x ; \tau, \xi)\right\} .
\end{aligned}
$$


The product in the right-hand side of (3.30) equals $\frac{\Gamma\left(a_{0}\right)\left[\Gamma\left(\left(\nu_{0}+\nu_{1}\right) \beta\right)\right]^{m-1}}{\Gamma\left(a_{0}+(m-1)\left(\nu_{0}+\nu_{1}\right) \beta\right)}$. This implies the convergence of the series for $\mathcal{R}$. The estimate (3.27) follows from (3.28) and (3.30).

Let us consider the iterations (3.25) for $n=1$, with the estimates coming from the Levi method for this case.

Lemma 6. Let $n=1$. Suppose that the function $K(t, x ; \tau, \xi)$ is continuous on

$$
\mathcal{P}_{1}=\left\{(t, x ; \tau, \xi): x, \xi \in \mathbb{R}^{1}, x \neq \xi, 0 \leq \tau<t \leq T\right\}
$$

and satisfies the inequality

$$
|K(t, x ; \tau, \xi)| \leq A_{1}(t-\tau)^{\gamma \beta-\beta-1} \exp \{-\mu \rho(t, x ; \tau, \xi)\}
$$

where $A_{1}, \mu>0,0<\gamma, \beta<1$. Then the series $\mathcal{R}(t, x ; \tau, \xi)=\sum_{m=1}^{\infty} K_{m}(t, x ; \tau, \xi)$ converges absolutely and uniformly on $\mathcal{P}_{1} \cap\{(t, x ; \tau, \xi): t-\tau \geq \delta>0\}$, and

$$
|\mathcal{R}(t, x ; \tau, \xi)| \leq C(t-\tau)^{\gamma \beta-\beta-1} \exp \left\{-\mu^{*} \rho(t, x ; \tau, \xi)\right\}
$$

with some $\mu^{*} \in(0, \mu)$.

Proof. Using Lemma 2, we find for any $\varepsilon>0$ that

$$
\begin{array}{r}
\left|K_{2}(t, x ; \tau, \xi)\right| \leq A_{1}^{2} \int_{\tau}^{t}[(t-\lambda)(\lambda-\tau)]^{\gamma \beta-\beta-1} d \lambda \int_{-\infty}^{\infty} \exp \{\mu[\rho(t, x ; \lambda, y)+\rho(\lambda, y ; \tau, \xi)] d y \\
\leq A_{2}(\varepsilon)(t-\tau)^{2 \gamma \beta-\beta-1} \exp \{-\mu(1-\varepsilon) \rho(t, x ; \tau, \xi)\} .
\end{array}
$$

Repeating the procedure we obtain the inequality

$$
\left|K_{m}(t, x ; \tau, \xi)\right| \leq A_{m}(\varepsilon)(t-\tau)^{m \gamma \beta-\beta-1} \exp \{-\mu(1-(m-1) \varepsilon) \rho(t, x ; \tau, \xi)\} .
$$

Let $m^{*}=\left[\frac{1+\beta}{\beta \gamma}\right]+1$. Then

$$
\left|K_{m^{*}}(t, x ; \tau, \xi)\right| \leq A_{m^{*}}(\varepsilon) \exp \left\{-\mu\left(1-m^{*} \varepsilon\right) \rho(t, x ; \tau, \xi)\right\} .
$$

Setting $\varepsilon=\frac{1}{p m^{*}}, p>2, \mu^{*}=\mu\left(1-p^{-1}\right), A_{0}=\max _{1 \leq m \leq m^{*}} A_{m}(\varepsilon)$, we can rewrite (3.32) as

$$
\left|K_{m^{*}}(t, x ; \tau, \xi)\right| \leq A_{0} \exp \left\{-\mu^{*} \rho(t, x ; \tau, \xi)\right\} .
$$

Now, for $m>m^{*}$, we proceed in a different way, in order to preserve the exponential factor $\mu^{*}$ in the estimates of further iterated kernels. By Lemma 1,

$$
\begin{aligned}
& \left|K_{m^{*}+1}(t, x ; \tau, \xi)\right| \leq A_{0}^{2} \int_{\tau}^{t}(t-\lambda)^{\gamma \beta-\beta-1} d \lambda \int_{-\infty}^{\infty} \exp \left\{\mu\left[\rho(t, x ; \lambda, y)+\left(1-p^{-1}\right) \rho(\lambda, y ; \tau, \xi)\right] d y\right. \\
& \leq A_{0}^{2} F \exp \left\{-\mu^{*} \rho(t, x ; \tau, \xi)\right\} \int_{\tau}^{t}(t-\lambda)^{\gamma \beta-1} d \lambda=A_{0}^{2} F B(\gamma \beta, 1)(t-\tau)^{\gamma \beta} \exp \left\{-\mu^{*} \rho(t, x ; \tau, \xi)\right\},
\end{aligned}
$$


where $F=\int_{0}^{\infty} \exp \left\{-\frac{\mu}{p}|y|^{\frac{1}{1-\beta}}\right\} d y$.

Similarly, we prove by induction that

$$
\left|K_{m^{*}+j}(t, x ; \tau, \xi)\right| \leq A_{0}\left(A_{0} F\right)^{j}(t-\tau)^{\gamma \beta j}\left\{\prod_{s=0}^{j-1} B(\gamma \beta, 1+s \gamma \beta)\right\} \exp \left\{-\mu^{*} \rho(t, x ; \tau, \xi)\right\} .
$$

Using the identity

$$
\prod_{s=0}^{j-1} B(\gamma \beta, 1+s \gamma \beta)=\frac{[\Gamma(\gamma \beta)]^{j}}{\Gamma(1+\gamma \beta j)}
$$

we see that the series for $\mathcal{R}$ is majorized by the convergent series

$$
\left\{\sum_{m=1}^{m^{*}} A_{m}(t-\tau)^{\gamma m \beta-\beta-1}+A_{0} \sum_{j=1}^{\infty} \frac{\left[\Gamma(\gamma \beta) A_{0} F\right]^{j}}{\Gamma(1+\gamma \beta j)}(t-\tau)^{\gamma \beta j}\right\} \exp \left\{-\mu^{*} \rho(t, x ; \tau, \xi)\right\} .
$$

This implies (3.31).

\section{PARAMETRIX}

4.1. The function $Z_{0}$. The parametrix kernel $Z_{0}(t, x-\xi, \zeta)$ is defined by the formula $(2.3)$ where $\left(A^{(i j)}\right)$ is the matrix inverse to the matrix $\left(a_{i j}(\zeta)\right)$ of the leading coefficients "frozen" at the parametric point $\zeta \in \mathbb{R}^{n}$. The estimates of $Z_{0}$ proved in [13] for the case of the constant coefficients $a_{i j}$ and collected in Proposition 1, remain valid for the parametrix, with the constants independent on $\zeta$.

Since properties of $Z_{0}$ are different for $n \geq 2$ and $n=1$, it is convenient to treat these cases separately. Therefore we assume in this and the next sections that $n \geq 2$. The case $n=1$ will be considered in Sect. 6 .

The behavior of $Z_{0}$ for $R \geq 1$ and $R<1$ is described in Proposition 1 separately. However it is possible to write equivalent unified estimates:

$$
\begin{gathered}
\left|D_{x}^{m} Z_{0}(t, x-\xi ; \zeta)\right| \leq C t^{-\alpha}|x-\xi|^{-n+2-|m|} \exp \{-\sigma \rho(t, x ; 0, \xi)\}, \quad|m| \leq 3, \\
\left|\mathbb{D}_{t}^{(\alpha)} Z_{0}(t, x-\xi ; \zeta)\right| \leq C t^{-\alpha}|x-\xi|^{-n} \exp \{-\sigma \rho(t, x ; 0, \xi)\}, \quad|m| \leq 3,
\end{gathered}
$$

where

$$
\rho(t, x ; \tau, \xi)=\left(\frac{|x-\xi|}{(t-\tau)^{\alpha / 2}}\right)^{\frac{2}{2-\alpha}}
$$

(this corresponds to the notation of Sect. 3.2 with $\beta=\alpha / 2$ ). If $n=2$, then (4.1) is valid only for $|m| \neq 0$. For the opposite case

$$
\left|Z_{0}(t, x-\xi ; \zeta)\right| \leq C t^{-\alpha}\left[\left|\log \left(t^{-\alpha}|x-\xi|^{2}\right)\right|+1\right] \exp \{-\sigma \rho(t, x ; 0, \xi)\} .
$$

The constants $C, \sigma>0$ in (4.1)-(4.3) do not depend on $\zeta$. Of course, the constants may be different from the ones in (2.5)-(2.10).

The next proposition gives similar estimates for differences of values of $Z_{0}$ and its derivatives corresponding to different values of the parameter $\zeta$. 
Proposition 3. For any $y, \zeta^{\prime}, \zeta^{\prime \prime} \in \mathbb{R}^{n}, 0<t \leq T$,

$$
\begin{aligned}
& \left|D_{x}^{m} Z_{0}\left(t, y ; \zeta^{\prime}\right)-D_{x}^{m} Z_{0}\left(t, y ; \zeta^{\prime \prime}\right)\right| \\
& \quad \leq C t^{-\alpha}\left|\zeta^{\prime}-\zeta^{\prime \prime}\right|^{\gamma}|y|^{-n+2-|m|} \exp \left\{-\sigma\left(t^{-\alpha / 2}|y|\right)^{\frac{2}{2-\alpha}}\right\}, \quad|m| \leq 2 .
\end{aligned}
$$

Proof. Let $m=0$. Denote

$$
\mathfrak{A}(y, \zeta)=\sum_{i, j=1}^{n} A^{(i j)}(\zeta) y_{i} y_{j}
$$

By our assumptions

$$
\begin{gathered}
C_{1}|y|^{2} \leq \mathfrak{A}(y, \zeta) \leq C_{2}|y|^{2} \\
\left|\mathfrak{A}\left(y, \zeta^{\prime}\right)-\mathfrak{A}\left(y, \zeta^{\prime \prime}\right)\right| \leq C\left|\zeta^{\prime}-\zeta^{\prime \prime}\right|^{\gamma}|y|^{2} \\
\left|\left[\operatorname{det} A\left(\zeta^{\prime}\right)\right]^{1 / 2}-\left[\operatorname{det} A\left(\zeta^{\prime \prime}\right)\right]^{1 / 2}\right| \leq C\left|\zeta^{\prime}-\zeta^{\prime \prime}\right|^{\gamma}
\end{gathered}
$$

According to (2.3), we have to use the estimate (3.8) for the function $H_{12}^{20}$, and also to find an estimate for a difference of such functions. Let

$$
\varphi(s)=s^{-n / 2} H_{12}^{20}\left[\omega s \mid \begin{array}{ll}
(1, \alpha) \\
\left(\frac{n}{2}, 1\right), & (1,1)
\end{array}\right], \quad \omega=\frac{1}{4} t^{-\alpha} .
$$

By (3.1),

$$
\varphi^{\prime}(s)=-\frac{n}{2} s^{-\frac{n}{2}-1} H_{12}^{20}\left[\omega s \mid \begin{array}{ll}
(1, \alpha) & \\
\left(\frac{n}{2}, 1\right), & (1,1)
\end{array}\right]-s^{-\frac{n}{2}-1} H_{23}^{30}\left[\omega s \mid \begin{array}{lll}
(1, \alpha), & (0,1) & \\
\left(\frac{n}{2}, 1\right), & (1,1), & (1,1)
\end{array}\right] .
$$

For large $s,(3.7)$ and (3.8) give the estimate

$$
\left|\varphi^{\prime}(s)\right| \leq C s^{\frac{n+2}{2(2-\alpha)}-\frac{n}{2}-1} \omega^{\frac{n+2}{2(2-\alpha)}} \exp \left(-\sigma|\omega|^{\frac{1}{2-\alpha}}\right),
$$

which implies the bound

$$
\left|\varphi\left(\mathfrak{A}\left(y, \zeta^{\prime}\right)\right)-\varphi\left(\mathfrak{A}\left(y, \zeta^{\prime \prime}\right)\right)\right| \leq C t^{-\frac{n \alpha}{2}}\left|\zeta^{\prime}-\zeta^{\prime \prime}\right|^{\gamma} \exp \left(-\sigma t^{-\frac{\alpha}{2-\alpha}}|y|^{\frac{2}{2-\alpha}}\right) .
$$

For small $s$, we have

$$
\left|\varphi^{\prime}(s)\right| \leq C \omega s^{-\frac{n}{2}}
$$

by (3.10) and (3.11); note that the logarithmic terms emerging for $n=2$ are cancelled.

As a result, we find that

$$
\left|Z_{0}\left(t, x-\xi ; \zeta^{\prime}\right)-Z_{0}\left(t, x-\xi, \zeta^{\prime \prime}\right)\right| \leq \begin{cases}C\left|\zeta^{\prime}-\zeta^{\prime \prime}\right|^{\gamma} t^{-\frac{n \alpha}{2}} \exp \left(-\sigma t^{-\frac{\alpha}{2-\alpha}}|y|^{\frac{2}{2-\alpha}}\right) & \text { if } t^{-\alpha}|y| \geq 1, \\ C\left|\zeta^{\prime}-\zeta^{\prime \prime}\right|^{\gamma} t^{-\alpha}|y|^{-n+2}, & \text { if } t^{-\alpha}|y| \leq 1,\end{cases}
$$

which is equivalent to the estimate (4.4) with $m=0$.

For the first and second derivatives the proof is similar, though somewhat cumbersome one has to use the asymptotics (3.7)-(3.12) for the H-functions; again for $n=2$ the logarithmic terms are cancelled. 
4.2. The function $Y_{0}$. The estimates for the function $Y_{0}$ are given in Proposition 2 for the case of constant coefficients. They carry over to the kernel $Y_{0}(t-\lambda, x-\xi ; \zeta)$ defined by $(2.4)$ with the coefficients $A^{(i j)}$ depending on $\zeta$ as above. The proof is a direct consequence of the formula (2.4) and properties of H-functions, in particular the differentiation formulas (3.1), (3.2) etc, the asymptotic relations (3.7) and (3.14)-(3.17). The calculations are simple but tedious, especially for $n \leq 4$, since we have to take into account several terms of the asymptotics of $\mathrm{H}$-functions near the origin.

Just as for the function $Z_{0}$, it is desirable to obtain unified estimates of $Y_{0}$ and its derivatives which are valid for all values of the independent variables. Here the problem is a little more complicated because the behavior of $Y_{0}$ is different for different values of the dimension $n$.

In particular, for $n=2$ we have

$$
\begin{aligned}
& \left|D_{x}^{m} Y_{0}(t-\tau, x-\xi, \zeta)\right| \leq C(t-\tau)^{-\frac{\alpha}{2}(2+|m|)+\alpha-1} \exp \{-\sigma \rho(t, x ; \tau, \xi)\}, \quad|m|=0,1 ; \\
& \left|D_{x}^{m} Y_{0}(t-\tau, x-\xi ; \zeta)\right| \\
& \quad \leq C(t-\tau)^{-\alpha-1}|x-\xi|^{2-|m|}\left[\left|\log \frac{|x-\xi|^{2}}{(t-\tau)^{\alpha}}\right|+1\right] \exp \{-\sigma \rho(t, x ; \tau, \xi)\}, \quad|m|=2,3 .
\end{aligned}
$$

These estimates follow immediately from (2.11), (2.21)-(2.24).

For $n=3$,

$$
\left|D_{x}^{m} Y_{0}(t-\tau, x-\xi ; \zeta)\right| \leq C(t-\tau)^{-\frac{\alpha}{2}(3+|m|)+\alpha-1} \exp \{-\sigma \rho(t, x ; \tau, \xi)\}, \quad|m|=0,1,
$$

and

$$
\left|D_{x}^{m} Y_{0}(t-\tau, x-\xi ; \zeta)\right| \leq C(t-\tau)^{-\alpha-1}|x-\xi|^{1-|m|} \exp \{-\sigma \rho(t, x ; \tau, \xi)\}, \quad|m|=2,3 .
$$

Similarly, for $n=4$,

$$
\begin{aligned}
& \left|D_{x}^{m} Y_{0}(t-\tau, x-\xi ; \zeta)\right| \\
& \quad \leq C(t-\tau)^{-\alpha-\frac{|m| \alpha}{2}-1}\left[\left|\log \frac{|x-\xi|^{2}}{(t-\tau)^{\alpha}}\right|+1\right] \exp \{-\sigma \rho(t, x ; \tau, \xi)\}, \quad|m|=0,1,2, \\
& \quad \leq D_{x}^{m} Y_{0}(t-\tau, x-\xi ; \zeta) \mid \\
& \quad \leq C(t-\tau)^{-2 \alpha-1}|x-\xi|^{-1}\left[\left|\log \frac{|x-\xi|^{2}}{(t-\tau)^{\alpha}}\right|+1\right] \exp \{-\sigma \rho(t, x ; \tau, \xi)\}, \quad|m|=3 .
\end{aligned}
$$

Finally, if $n>4$, then

$$
\left|D_{x}^{m} Y_{0}(t-\tau, x-\xi ; \zeta)\right| \leq C(t-\tau)^{-\alpha-1}|x-\xi|^{-n-|m|+4} \exp \{-\sigma \rho(t, x ; \tau, \xi)\}, \quad|m| \leq 3 .
$$

Just as in Proposition 3, the estimates for the differences $D_{x}^{m} Y_{0}\left(t-\tau, x-\xi ; \zeta^{\prime}\right)-D_{x}^{m} Y_{0}(t-$ $\left.\tau, x-\xi ; \zeta^{\prime \prime}\right)$ have the following form: the right-hand sides of (4.5)-(4.11) are multiplied by $\left|\zeta^{\prime}-\zeta^{\prime \prime}\right|^{\gamma}$. For example, if $n>4$, then

$$
\begin{aligned}
& \mid D_{x}^{m} Y_{0}(t-\tau, \\
& \left.\quad \leq-\xi ; \zeta^{\prime}\right)-D_{x}^{m} Y_{0}\left(t-\tau, x-\xi ; \zeta^{\prime \prime}\right) \mid \\
& \quad \leq C(t-\tau)^{-\alpha-1}\left|\zeta^{\prime}-\zeta^{\prime \prime}\right|^{\gamma}|x-\xi|^{-n-|m|+4} \exp \{-\sigma \rho(t, x ; \tau, \xi)\}, \quad|m| \leq 3 .
\end{aligned}
$$


4.3. Integral identities. It follows from the construction of the function $Z_{0}$ that

$$
\int_{\mathbb{R}^{n}} Z_{0}(t, x-\xi ; \zeta) d \xi=1
$$

(see [13]). Next, from (2.4) we see that

$$
\begin{aligned}
& \int_{\mathbb{R}^{n}} Y_{0}(t-\tau, x-\xi ; \zeta) d \xi=(t-\tau)^{\alpha-1} \pi^{-n / 2} \int_{\mathbb{R}^{n}}|y|^{-n} H_{12}^{20}\left[\begin{array}{l|l}
1 & \frac{1}{4}(t-\tau)^{-\alpha}|y|^{2}
\end{array} \mid \begin{array}{ll}
(\alpha, \alpha) \\
\left(\frac{n}{2}, 1\right), & (1,1)
\end{array}\right] d y \\
& =C(t-\tau)^{\alpha-1} \int_{0}^{\infty} r^{-1} H_{12}^{20}\left[\frac{1}{4}(t-\tau)^{-\alpha} r^{2} \mid \begin{array}{ll}
(\alpha, \alpha) & \\
\left(\frac{n}{2}, 1\right), & (1,1)
\end{array}\right] d r \\
& =C_{1}(t-\tau)^{\alpha-1} \int_{0}^{\infty} s^{-1} H_{12}^{20}\left[\frac{1}{4}(t-\tau)^{-\alpha} s \mid \begin{array}{ll}
(\alpha, \alpha) & \\
\left(\frac{n}{2}, 1\right), & (1,1)
\end{array}\right] d s
\end{aligned}
$$

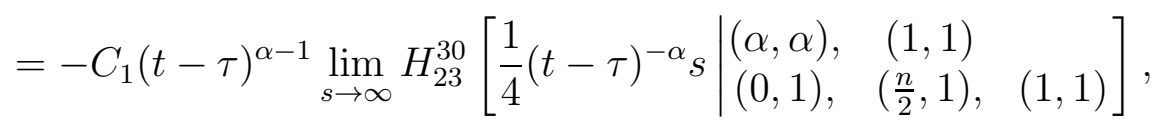

so that

$$
\int_{\mathbb{R}^{n}} Y_{0}(t-\tau, x-\xi, \zeta) d \xi=0
$$

We have used the integration formula

$$
\int_{0}^{t} s^{-1} H_{12}^{20}\left[a s \mid \begin{array}{ll}
(\alpha, \alpha) \\
\left(\frac{n}{2}, 1\right), & (1,1)
\end{array}\right] d s=H_{23}^{21}\left[a t \mid \begin{array}{lll}
(1,1), & (\alpha, \alpha) & \\
\left(\frac{n}{2}, 1\right), & (1,1), & (0,1)
\end{array}\right]
$$

([20], formula 1.16.4.1), the identity connecting $H_{23}^{21}$ and $H_{23}^{30}$ ([20], 8.3.2.9), and the asymptotics (3.7) of $H_{23}^{30}$.

4.4. Further estimates. Below we will need also the first time derivative of the function $Z_{0}(t, x ; \zeta)$. We find from $(2.3)$ and $(3.1)$ that

$$
\begin{aligned}
\frac{\partial Z_{0}(t, x ; \zeta)}{\partial t}=\frac{\alpha \pi^{-n / 2} t^{-1}}{(\operatorname{det} A)^{1 / 2}}\left[\sum_{i, j=1}^{n} A^{(i j)} x_{i} x_{j}\right. & \\
& \times H_{23}^{30}\left[\frac{1}{4} t^{-\alpha} \sum_{i, j=1}^{n} A^{(i j)} x_{i} x_{j} \mid \begin{array}{lll}
(1, \alpha), & (0,1) \\
\left(\frac{n}{2}, 1\right), & (1,1), & (1,1)
\end{array}\right] .
\end{aligned}
$$

Using the asymptotics (3.7) and (3.11) of the function $H_{23}^{30}$ we can obtain an estimate of $\frac{\partial Z_{0}}{\partial t}$. For example, if $n \geq 3$, then

$$
\left|\frac{\partial Z_{0}(t, x ; \zeta)}{\partial t}\right| \leq \begin{cases}C t^{-1-\frac{\alpha n}{2}} \exp \left\{-\sigma\left(t^{-\alpha / 2}|x|\right)^{\frac{2}{2-\alpha}}\right\}, & \text { if } t^{-\alpha / 2}|x| \geq 1 \\ C t^{-\alpha-1}|x|^{-n+2}, & \text { if } t^{-\alpha / 2}|x|<1\end{cases}
$$


We can also use a roughened unified estimate

$$
\left|\frac{\partial Z_{0}(t, x ; \zeta)}{\partial t}\right| \leq C t^{-1}|x|^{-n} \exp \left\{-\sigma\left(t^{-\alpha / 2}|x|\right)^{\frac{2}{2-\alpha}}\right\} .
$$

As before, we get also an estimate for the difference $\left|\frac{\partial Z_{0}\left(t, x ; \zeta^{\prime}\right)}{\partial t}-\frac{\partial Z_{0}\left(t, x ; \zeta^{\prime \prime}\right)}{\partial t}\right|$ whose upper bound is the expression in the right-hand side of (4.15) or $\left(4.15^{\prime}\right)$ multiplied by $\left|\zeta^{\prime}-\zeta^{\prime \prime}\right|^{\gamma}$. Since

$$
\int_{\mathbb{R}^{n}} \frac{\partial Z_{0}\left(t, x-\xi ; \zeta^{\prime}\right)}{\partial t} d \xi=0
$$

by virtue of (4.13), the above estimates imply also the estimate

$$
\left|\int_{\mathbb{R}^{n}} \frac{\partial Z_{0}(t, x-\xi ; \xi)}{\partial t} d \xi\right| \leq C t^{-1+\frac{\alpha \gamma}{2}}
$$

obtained by subtracting the (zero) integral of $\frac{\partial Z_{0}(t, x ; x)}{\partial t}$ and using the estimate for the difference of the derivatives.

\section{THE LEVI METHOD $(n \geq 2)$}

5.1. The scheme. We look for the functions $Y, Z$ appearing in (1.4) assuming the following integral representations:

$$
\begin{gathered}
Z(t, x ; \xi)=Z_{0}(t, x-\xi ; \xi)+\int_{0}^{t} d \lambda \int_{\mathbb{R}^{n}} Y_{0}(t-\lambda, x-y ; y) Q(\lambda, y ; \xi) d y ; \\
Y(t, x ; \xi)=Y_{0}(t-\tau, x-\xi ; \xi)+\int_{\tau}^{t} d \lambda \int_{\mathbb{R}^{n}} Y_{0}(t-\lambda, x-y ; y) \Psi(\lambda, y ; \xi) d y ;
\end{gathered}
$$

the functions $Z_{0}, Y_{0}$ were examined in detail in Sect. 4.

For the functions $Q$ and $\Psi$ we assume the integral equations

$$
\begin{aligned}
& Q(t, x ; \xi)=M(t, x ; \xi)+\int_{0}^{t} d \lambda \int_{\mathbb{R}^{n}} K(t-\lambda, x ; y) Q(\lambda, y ; \xi) d y, \\
& \Psi(t, x ; \xi)=K(t, x ; \xi)+\int_{\tau}^{t} d \lambda \int_{\mathbb{R}^{n}} K(t-\lambda, x ; y) \Psi(\lambda, y ; \xi) d y,
\end{aligned}
$$


where

$$
\begin{aligned}
& M(t, x ; \xi)=\sum_{i, j=1}^{n}\left\{\left[a_{i j}(x)-a_{i j}(\xi)\right] \frac{\partial^{2}}{\partial x_{i} \partial x_{j}} Z_{0}(t, x-\xi ; \xi)\right\} \\
&+\sum_{i=1}^{n} b_{i}(x) \frac{\partial Z_{0}(t, x-\xi ; \xi)}{\partial x_{i}}+c(x) Z_{0}(t, x-\xi ; \xi), \\
& K(t, x ; \xi)=\sum_{i, j=1}^{n}\left\{\left[a_{i j}(x)-a_{i j}(\xi)\right] \frac{\partial^{2}}{\partial x_{i} \partial x_{j}} Y_{0}(t, x-\xi ; \xi)\right\} \\
&+\sum_{i=1}^{n} b_{i}(x) \frac{\partial Y_{0}(t, x-\xi ; \xi)}{\partial x_{i}}+c(x) Y_{0}(t, x-\xi ; \xi) .
\end{aligned}
$$

Using the estimates (4.1)-(4.3) we find that

$$
|M(t, x ; \xi)| \leq C t^{-\alpha}|x-\xi|^{-n+\gamma} \exp \{-\sigma \rho(t, x ; 0, \xi)\} .
$$

In order to obtain estimates for $K$, we have to use estimates for $Y_{0}$, different for different values of $n$, and for different domains, and then to roughen the resulting estimates into less exact but unified bounds convenient for the Levi method.

Proposition 4. For any $n \geq 2$

$$
|K(t, x ; \xi)| \leq C t^{(\gamma-\eta) \frac{\alpha}{2}-1}|x-\xi|^{-n+\eta} \exp \{-\sigma \rho(t, x ; 0, \xi)\},
$$

with $0<\eta<\gamma$.

Proof. Using Proposition 2 we write the estimate for $|x-\xi| \geq t^{\alpha / 2}$ :

$$
|K(t, x ; \xi)| \leq C t^{-\frac{\alpha n}{2}+\frac{\alpha \gamma}{2}-1} \exp \{-\sigma \rho(t, x ; 0, \xi)\} .
$$

For $|x-\xi| \leq t^{\alpha / 2}$ we consider various cases separately. If $n=3$, or $n>4$, then

$$
|K(t, x ; \xi)| \leq C t^{-\alpha+\frac{\alpha \gamma}{2}-1}|x-\xi|^{-n+2} \exp \{-\sigma \rho(t, x ; 0, \xi)\} .
$$

Since

$$
|x-\xi|^{2}=|x-\xi|^{\eta}|x-\xi|^{2-\eta} \leq|x-\xi|^{\eta} t^{\frac{\alpha}{2}(2-\eta)},
$$

(5.7) and (5.8) imply (5.6). Note that transforming (5.7) we change the constant $\sigma$.

If $n=2$ or $n=4$, the initial bound for $K$ is

$$
|K(t, x ; \xi)| \leq C t^{-\frac{\alpha n}{2}+\frac{\alpha \gamma}{2}-1}\left(\left|\log \frac{|x-\xi|^{2}}{t^{\alpha}}\right|+1\right) \exp \{-\sigma \rho(t, x ; 0, \xi)\} .
$$

We roughen the estimate (5.9) replacing the factor $\left|\log \frac{|x-\xi|^{2}}{t^{\alpha}}\right|+1$ with the factor $\left(\frac{|x-\xi|}{t^{\alpha / 2}}\right)^{-n+\eta}$, which results in (5.6). 
Next we have to study the increments

$$
\begin{aligned}
\Delta_{x} M(t, x ; \xi) & =M(t, x ; \xi)-M\left(t, x^{\prime} ; \xi\right), \\
\Delta_{x} K(t, x ; \xi) & =K(t, x ; \xi)-K\left(t, x^{\prime} ; \xi\right),
\end{aligned}
$$

$x, x^{\prime} \in \mathbb{R}^{n}$.

We can write $\Delta_{x} M(t, x ; \xi)=M_{1}+M_{2}+M_{3}$ where

$$
\begin{gathered}
M_{1}=\sum_{i, j=1}^{n} \Delta_{x} a_{i j}(x) \frac{\partial^{2} Z_{0}(t, x-\xi ; \xi)}{\partial x_{i} \partial x_{j}}, \\
M_{2}=\sum_{i, j=1}^{n}\left[a_{i j}\left(x^{\prime}\right)-a_{i j}(\xi)\right] \Delta_{x} \frac{\partial^{2} Z_{0}\left(t, x^{\prime}-\xi ; \xi\right)}{\partial x_{i}^{\prime} \partial x_{j}^{\prime}},
\end{gathered}
$$

and the term $M_{3}$ contains lower order derivatives. Since $M_{3}$ has a weaker singularity and does not influence the estimates, we omit its detailed description and consider only $M_{1}$ and $M_{2}$.

An estimate for $M_{1}$ is given directly:

$$
\left|M_{1}\right| \leq C_{1}\left|x-x^{\prime}\right|^{\gamma} t^{-\alpha}|x-\xi|^{-n} \exp \{-\sigma \rho(t, x ; 0, \xi)\} .
$$

Using (4.1) we find that

$$
\begin{aligned}
& \quad\left|\Delta_{x} \frac{\partial^{2} Z_{0}\left(t, x^{\prime}-\xi ; \xi\right)}{\partial x_{i}^{\prime} \partial x_{j}^{\prime}}\right| \leq\left|\Delta_{x} \frac{\partial^{2} Z_{0}}{\partial x_{i}^{\prime} \partial x_{j}^{\prime}}\right|^{\nu}\left(\left|\frac{\partial^{2} Z_{0}}{\partial x_{i} \partial x_{j}}\right|\right. \\
& \left.\leq\left|\frac{\partial^{2} Z_{0}}{\partial x_{i}^{\prime} \partial x_{j}^{\prime}}\right|\right)^{1-\nu} \\
& \leq C_{3}^{\nu} t^{-\alpha \nu}|\widetilde{x}-\xi|^{-n \nu-\nu} \exp \{-\nu \sigma \rho(t, \widetilde{x} ; 0, \xi)\}\left|x-x^{\prime}\right|^{\nu} C_{2}^{1-\nu} t^{-\alpha(1-\nu)}\left[|x-\xi|^{-n} \exp \{-\sigma \rho(t, x ; 0, \xi)\}\right. \\
& \left.+\left|x^{\prime}-\xi\right|^{-n} \exp \left\{-\sigma \rho\left(t, x^{\prime} ; 0, \xi\right)\right\}\right]^{1-\nu}
\end{aligned}
$$

where $\widetilde{x}=x+\theta\left(x^{\prime}-x\right), 0<\theta<1,0<\nu<\gamma$, and we assumed that $\min \left\{|x-\xi|,\left|x^{\prime}-\xi\right|\right\} \neq 0$. From (5.10) and (5.11) we find that

$$
\left|\Delta_{x} M(t, x ; \xi)\right| \leq C_{4} t^{-\alpha}\left[\left|x-x^{\prime}\right|^{\gamma}|x-\xi|^{-n} \exp \{-\sigma \rho(t, x ; 0, \xi)\}+\left|x-x^{\prime}\right|^{\nu}\left|x^{\prime}-\xi\right|^{\gamma} \widetilde{M}(t, x ; \xi)\right]
$$

where

$$
\begin{aligned}
\widetilde{M}(t, x ; \xi)=|\widetilde{x}-\xi|^{-n \nu-\nu} \exp \{-\nu \sigma \rho(t, \widetilde{x} ; 0, \xi)\}\left[|x-\xi|^{-n} \exp \{-\sigma \rho(t, x ; 0, \xi)\}\right. \\
\left.+\left|x^{\prime}-\xi\right|^{-n} \exp \left\{-\sigma \rho\left(t, x^{\prime} ; 0, \xi\right)\right\}\right]^{1-\nu}
\end{aligned}
$$

We will use also the following estimate which is a direct consequence of the definition of $\Delta_{x} M$ :

$$
\begin{aligned}
\left|\Delta_{x} M(t, x ; \xi)\right| \leq C t^{-\alpha}\left(|x-\xi|^{-n+\gamma} \exp \{-\sigma \rho(t,\right. & ; 0, \xi)\} \\
& \left.+\left|x^{\prime}-\xi\right|^{-n+\gamma} \exp \left\{-\sigma \rho\left(t, x^{\prime} ; 0, \xi\right)\right\}\right) .
\end{aligned}
$$

Let us consider two possible cases. 
a) Suppose that $\left|x-x^{\prime}\right|>A t^{\alpha / 2}$. It follows from (5.13) that

$$
\begin{aligned}
\left|\Delta_{x} M(t, x ; \xi)\right| \leq C_{5}(A) t^{(\gamma-\varepsilon) \frac{\alpha}{2}-\alpha}\left[|x-\xi|^{-n+\varepsilon} \exp \left\{-\sigma_{1} \rho(t, x ; 0, \xi)\right\}\right. & \\
& \left.+\left|x^{\prime}-\xi\right|^{-n+\varepsilon} \exp \left\{-\sigma_{1} \rho\left(t, x^{\prime} ; 0, \xi\right)\right\}\right], \quad \varepsilon>0
\end{aligned}
$$

whence

$$
\begin{aligned}
\left|\Delta_{x} M(t, x ; \xi)\right| \leq C_{6} t^{-\alpha}\left|x-x^{\prime}\right|^{\gamma-\varepsilon}\left[|x-\xi|^{-n+\varepsilon} \exp \left\{-\sigma_{1} \rho(t, x ; 0, \xi)\right\}\right. \\
\left.+\left|x^{\prime}-\xi\right|^{-n+\varepsilon} \exp \left\{-\sigma_{1} \rho\left(t, x^{\prime} ; 0, \xi\right)\right\}\right], \quad \varepsilon>0
\end{aligned}
$$

b) Let $\left|x-x^{\prime}\right| \leq A t^{\alpha / 2}$ with some $A>0$. If $\left|x-x^{\prime}\right|>\eta \max \left(|\xi-x|,\left|\xi-x^{\prime}\right|\right)$ with some $\eta>0$, then we obtain again the estimate (5.14). Thus, we have now to consider the most complicated subcase, for which

$$
\left|x-x^{\prime}\right| \leq \eta \max \left(|\xi-x|,\left|\xi-x^{\prime}\right|\right), \quad \eta>0
$$

Here we use the estimate (5.11).

Without restricting generality, we may assume that $\left|\xi-x^{\prime}\right| \leq|\xi-x|$. Then $\left|x-x^{\prime}\right| \leq \eta|\xi-x|$,

$$
|\widetilde{x}-\xi|=\left|x-\xi+\theta\left(x^{\prime}-x\right)\right| \geq|x-\xi|-\theta \eta|x-\xi| \geq(1-\eta)|x-\xi| .
$$

Note that the function

$$
r \mapsto r^{-n} \exp \left\{-\sigma\left(\frac{r}{t^{\alpha / 2}}\right)^{\frac{2}{2-\alpha}}\right\}, \quad 0<\alpha<1,
$$

is monotone decreasing. This implies the estimate

$$
|\widetilde{M}(t, x ; \xi)| \leq C_{7}\left[|x-\xi|^{-n-\nu} \exp \left\{-\sigma_{1} \rho(t, x ; 0, \xi)\right\}+\left|x^{\prime}-\xi\right|^{-n-\nu} \exp \left\{-\sigma \rho\left(t, x^{\prime} ; 0, \xi\right)\right\}\right] .
$$

Setting $\varepsilon=\gamma-\nu$ and using (5.13), (5.14), we come to the estimate contained in the following proposition.

Proposition 5. If $x^{\prime \prime}$ is one of the points $x, x^{\prime}$ for which $\left|x^{\prime \prime}-\xi\right|=\min \left\{|x-\xi|,\left|x^{\prime}-\xi\right|\right\}$, then

$$
\begin{aligned}
& \left|\Delta_{x} M(t, x ; \xi)\right| \leq C t^{-\alpha}\left|x-x^{\prime}\right|^{\gamma-\varepsilon}\left|x^{\prime \prime}-\xi\right|^{-n+\varepsilon} \exp \left\{-\sigma \rho\left(t, x^{\prime \prime} ; 0, \xi\right)\right\} \\
& \left|\Delta_{x} K(t, x ; \xi)\right| \leq C t^{-1}\left|x-x^{\prime}\right|^{\gamma-\varepsilon}\left|x^{\prime \prime}-\xi\right|^{-n+\varepsilon} \exp \left\{-\sigma \rho\left(t, x^{\prime \prime} ; 0, \xi\right)\right\} .
\end{aligned}
$$

The proof of (5.16) is similar to that of (5.15), since the estimates for $Y_{0}$ have a structure similar to those for $Z_{0}$.

Now we are ready to consider the integral equations (5.3) and (5.4).

Proposition 6. The integral equations (5.3) and (5.4) have the solutions $Q(t, x ; \xi), \Psi(t, x ; \xi)$, which are continuous for $x \neq \xi$ and satisfy the estimates

$$
|Q(t, x ; \xi)| \leq C t^{-\alpha}|x-\xi|^{-n+\gamma} \exp \{-\sigma \rho(t, x ; 0, \xi)\},
$$




$$
\begin{gathered}
\left|\Delta_{x} Q(t, x ; \xi)\right| \leq C t^{-\alpha}\left|x-x^{\prime}\right|^{\gamma-\varepsilon}\left|x^{\prime \prime}-\xi\right|^{-n+\varepsilon} \exp \left\{-\sigma \rho\left(t, x^{\prime \prime} ; 0, \xi\right)\right\} \\
|\Psi(t, x ; \xi)| \leq C t^{-1}|x-\xi|^{-n+\gamma} \exp \{-\sigma \rho(t, x ; 0, \xi)\} \\
\left|\Delta_{x} \Psi(t, x ; \xi)\right| \leq C t^{-1}\left|x-x^{\prime}\right|^{\gamma-\varepsilon}\left|x^{\prime \prime}-\xi\right|^{-n+\varepsilon} \exp \left\{-\sigma \rho\left(t, x^{\prime \prime} ; 0, \xi\right)\right\}
\end{gathered}
$$

with an arbitrary $\varepsilon \in(0, \gamma)$ and the constants depending on $\varepsilon$. If $n=2$ or $n=4$, then for any $\mu>0$

$$
\begin{gathered}
|Q(t, x ; \xi)| \leq C t^{-\alpha-\frac{\mu \alpha}{2}}|x-\xi|^{-n+\varepsilon-\mu} \exp \{-\sigma \rho(t, x ; 0, \xi)\} \\
\left|\Delta_{x} Q(t, x ; \xi)\right| \leq C t^{-\alpha-\frac{\mu \alpha}{2}}\left|x-x^{\prime}\right|^{\gamma-\varepsilon}\left|x^{\prime \prime}-\xi\right|^{-n+\varepsilon-\mu} \exp \left\{-\sigma \rho\left(t, x^{\prime \prime} ; 0, \xi\right)\right\} \\
|\Psi(t, x ; \xi)| \leq C t^{-1-\frac{\mu \alpha}{2}}|x-\xi|^{-n+\varepsilon-\mu} \exp \{-\sigma \rho(t, x ; 0, \xi)\}, \\
\left|\Delta_{x} \Psi(t, x ; \xi)\right| \leq C t^{-1-\frac{\mu \alpha}{2}}\left|x-x^{\prime}\right|^{\gamma-\varepsilon}\left|x^{\prime \prime}-\xi\right|^{-n+\varepsilon-\mu} \exp \left\{-\sigma \rho\left(t, x^{\prime \prime} ; 0, \xi\right)\right\}
\end{gathered}
$$

Here the constants depend on $\varepsilon$ and $\mu$.

Proof. The equations (5.3) and (5.4) are solved by iterations. The estimates (5.17), (5.17'), (5.19), (5.19') follow from Lemma 5.

Let us prove (5.18). We have $\Delta_{x} Q=\Delta_{x} M+J$ where

$$
\begin{gathered}
J=\int_{0}^{t} d \lambda \int_{\mathbb{R}^{n}} \Delta_{x} K(t-\lambda, x ; y) Q(\lambda, y ; \xi) d y \\
|J| \leq C\left|x-x^{\prime}\right|^{\gamma-\varepsilon} \int_{0}^{t}(t-\lambda)^{-1} \lambda^{-\alpha} d \lambda\left[\int_{\mathbb{R}^{n}}|x-y|^{-n+\varepsilon}|y-\xi|^{-n+\gamma} \exp \{-\sigma \rho(t, x ; \lambda, y)\right. \\
\left.-\sigma \rho(\lambda, y ; 0, \xi)\} d y+\int_{\mathbb{R}^{n}}\left|x^{\prime}-y\right|^{-n+\varepsilon}|y-\xi|^{-n+\gamma} \exp \left\{-\sigma \rho\left(t, x^{\prime} ; \lambda, y\right)-\sigma \rho(\lambda, y ; 0, \xi)\right\} d y\right] \\
\leq C\left|x-x^{\prime}\right|^{\gamma-\varepsilon} \int_{0}^{t}(t-\lambda)^{-1+\frac{\alpha \varepsilon}{4}} \lambda^{(1-\alpha)-1} d \lambda\left[\int _ { \mathbb { R } ^ { n } } | x - y | ^ { - n + \frac { \varepsilon } { 2 } } | y - \xi | ^ { - n + \gamma } \operatorname { e x p } \left\{-\sigma_{1} \rho(t, x ; \lambda, y)\right.\right. \\
\left.\left.-\sigma_{1} \rho(\lambda, y ; 0, \xi)\right\} d y+\int_{\mathbb{R}^{n}}\left|x^{\prime}-y\right|^{-n+\frac{\varepsilon}{2}}|y-\xi|^{-n+\gamma} \exp \left\{-\sigma_{1} \rho\left(t, x^{\prime} ; \lambda, y\right)-\sigma_{1} \rho(\lambda, y ; 0, \xi)\right\} d y\right] .
\end{gathered}
$$

By Lemma 3, the integral (5.21) is estimated as follows:

$$
\begin{aligned}
|J| \leq C\left|x-x^{\prime}\right|^{\gamma-\varepsilon} t^{-\alpha+\frac{\alpha \varepsilon}{4}}\left[|x-\xi|^{-n+\gamma+\frac{\varepsilon}{2}} \exp \left\{-\sigma_{2} \rho(t, x ; 0, \xi)\right\}\right. & \\
& \left.+\left|x^{\prime}-\xi\right|^{-n+\gamma+\frac{\varepsilon}{2}} \exp \left\{-\sigma_{2} \rho(t, x ; 0, \xi)\right\}\right] .
\end{aligned}
$$


Together with (5.15), this implies (5.18) with an appropriate $\sigma>0$.

The proof of the inequalities $(5.20),\left(5.20^{\prime}\right)$ is similar.

5.2. Heat potentials. In order to verify that the functions (5.1) and (5.2) indeed solve the Cauchy problem via the representation (1.4), we have to study the heat potential

$$
W(t, x)=\int_{0}^{t} d \lambda \int_{\mathbb{R}^{n}} Y_{0}(t-\lambda, x-y ; y) f(\lambda, y) d y
$$

for two situations. Firstly, we must consider the case of a bounded, locally Hölder continuous (in $x$ ) function $f$, jointly continuous in $(t, x) \in[0, T] \times \mathbb{R}^{n}$. This is, of course, dictated by the formula (1.4) containing a term which solves the inhomogeneous equation with the zero initial condition. Note that $Y_{0}$ is the main singular part of the function $Y$ appearing in (1.4). Secondly, heat potentials appear also in (5.1) and (5.2), where the role of $f$ is played by the functions $Q$ and $\Psi$ which are much more singular (see Proposition 6).

Let us consider the potential (5.22) with a bounded, locally Hölder continuous $f$. The existence of the integral (5.22) and the possibility to find its first order derivatives in $x$ by differentiating under the integral's symbol follow directly from the estimates (4.5), (4.7), (4.9), and (4.11). For studying the second order derivatives we consider the function

$$
W(t, x)=\int_{0}^{t-h} d \lambda \int_{\mathbb{R}^{n}} Y_{0}(t-\lambda, x-y ; y) f(\lambda, y) d y, \quad h>0 .
$$

As $t-\lambda \geq h$, the function $Y_{0}$ and its first and second derivatives have integrable singularities at $x=y$. Therefore we may differentiate under the integral's symbol, so that

$$
\frac{\partial^{2} W_{h}(t, x)}{\partial x_{i} \partial x_{j}}=J_{1}+J_{2}
$$

where

$$
\begin{gathered}
J_{1}=\int_{0}^{t-h} d \lambda \int_{\mathbb{R}^{n}} \frac{\partial^{2} Y_{0}(t-\lambda, x-y ; y)}{\partial x_{i} \partial x_{j}}[f(\lambda, y)-f(\lambda, x)] d y, \\
J_{2}=\int_{0}^{t-h} f(\lambda, x) d \lambda \int_{\mathbb{R}^{n}} \frac{\partial^{2} Y_{0}(t-\lambda, x-y ; y)}{\partial x_{i} \partial x_{j}} d y .
\end{gathered}
$$

We decompose further, $J_{1}=J_{1}^{(1)}+J_{1}^{(2)}$, where $J_{1}^{(1)}$ corresponds to integration over the set $\Pi^{(1)}=\left\{y \in \mathbb{R}^{n}:|x-y| \leq(t-\lambda)^{\alpha / 2}\right\}$ while for $J_{1}^{(2)}$ the domain of integration is $\Pi^{(2)}=$ $\left\{y \in \mathbb{R}^{n}:|x-y|>(t-\lambda)^{\alpha / 2}\right\}$. Suppose that, for example, $n>4$; other cases are treated similarly. If $y \in \Pi^{(1)}$, then

$$
\left|\frac{\partial^{2} Y_{0}(t-\lambda, x-y ; y)}{\partial x_{i} \partial x_{j}}\right| \leq C(t-\lambda)^{-\alpha-1}|x-y|^{-n+2},
$$


so that the $\Pi^{(1)}$ part of the integral in (5.23) is majorized by

$$
I_{1}^{(1)}=C \int_{0}^{t-h}(t-\lambda)^{-\alpha-1} d \lambda \int_{\Pi^{(1)}}|x-y|^{-n+2+\gamma} d y,
$$

and the change of variables $\widehat{y}=(t-\lambda)^{-\alpha / 2}(y-x)$ gives

$$
I_{1}^{(1)} \leq C \int_{0}^{t-h}(t-\lambda)^{-1+\frac{\alpha \gamma}{2}} d \lambda \int_{|\widehat{y}| \leq 1}|\widehat{y}|^{-n+2+\gamma} d \widehat{y} .
$$

Here $\gamma>0$ is the Hölder exponent of the function $f(\lambda, x)$ in $x$.

For $y \in \Pi^{(2)}$ we use the inequality

$$
\left|\frac{\partial^{2} Y_{0}(t-\lambda, x-y ; y)}{\partial x_{i} \partial x_{j}}\right| \leq C(t-\lambda)^{-\frac{\alpha n}{2}-1} \exp \left\{-\sigma\left(\frac{|x-y|}{(t-\lambda)^{\alpha / 2}}\right)^{\frac{2}{2-\alpha}}\right\},
$$

a consequence of the general estimate (4.11). A similar argument shows that the $\Pi^{(2)}$ part of the integral in $J_{1}$ is majorized by

$$
C \int_{0}^{t-h}(t-\lambda)^{-1+\frac{\alpha \gamma}{2}} d \lambda \int_{|\widehat{y}|>1}|\widehat{y}|^{\gamma} \exp \left\{-\sigma|\widehat{y}|^{\frac{2}{2-\alpha}}\right\} d \widehat{y}
$$

We see the existence of the limit of $J_{1}$ as $h \rightarrow 0$.

Let us consider $J_{2}$. Using (4.14) we have

$$
J_{2}=\int_{0}^{t-h} f(\lambda, x) d \lambda \int_{\mathbb{R}^{n}}\left[\frac{\partial^{2} Y_{0}(t-\lambda, x-y ; y)}{\partial x_{i} \partial x_{j}}-\left.\frac{\partial^{2} Y_{0}(t-\lambda, x-y ; \delta)}{\partial x_{i} \partial x_{j}}\right|_{\delta=x}\right] d y .
$$

By (4.12), the expression in brackets is majorized by

$$
C|x-y|^{-n+2+\gamma}(t-\lambda)^{-\alpha-1} \exp \{-\sigma \rho(t, x ; \lambda, y)\} .
$$

The same change of variables (used above) shows that the integrand in (5.26) is majorized by

$$
(t-\lambda)^{-1+\frac{\alpha \gamma}{2}}|\widehat{y}|^{-n+2+\gamma} \exp \left\{-\sigma|\widehat{y}|^{\frac{2}{2-\alpha}}\right\} \text {. }
$$

This means the existence of the limit of $J_{2}$ as $h \rightarrow 0$. Simultaneously we have proved the formula

$$
\begin{aligned}
\frac{\partial^{2} W(t, x)}{\partial x_{i} \partial x_{j}}=\int_{0}^{t} d \lambda \int_{\mathbb{R}^{n}} \frac{\partial^{2} Y_{0}(t-\lambda, x-y ; y)}{\partial x_{i} \partial x_{j}}[f(\lambda, y)-f(\lambda, x)] d y \\
\\
+\int_{0}^{t} f(\lambda, x) d \lambda \int_{\mathbb{R}^{n}} \frac{\partial^{2} Y_{0}(t-\lambda, x-y ; y)}{\partial x_{i} \partial x_{j}} d y .
\end{aligned}
$$


Now we have to carry out a similar procedure for the potential (5.22) with $f(\lambda, y)=$ $Q(\lambda, y ; \xi)$ with a fixed $\xi$ (the case of $\Psi(\lambda, y ; \tau, \xi)$ is quite similar). We use the estimates $(5.17)$ and (5.18) for the function $Q$ and its increment, and also the following roughened estimate:

$$
\left|\frac{\partial^{2} Y_{0}(t-\lambda, x-y ; \xi)}{\partial x_{i} \partial x_{j}}\right| \leq C(t-\lambda)^{-1}|x-y|^{-n} \exp \{-\sigma \rho(t, x ; \lambda, y)\} .
$$

In fact we have to prove convergence of the integrals

$$
\begin{gathered}
I_{1}^{\prime}=\int_{0}^{t} d \lambda \int_{\mathbb{R}^{n}}\left|\frac{\partial^{2} Y_{0}(t-\lambda, x-y ; y)}{\partial x_{i} \partial x_{j}}\right| Q(\lambda, x ; \xi)-Q(\lambda, y ; \xi) \mid d y, \\
I_{2}^{\prime}=\int_{0}^{t}|Q(\lambda, x ; \xi)| d \lambda \int_{\mathbb{R}^{n}}\left|\frac{\partial^{2} Y_{0}(t-\lambda, x-y ; y)}{\partial x_{i} \partial x_{j}}-\frac{\partial^{2} Y_{0}(t-\lambda, x-y ; \delta)}{\partial x_{i} \partial x_{j}}\right|_{\delta=x} \mid d y .
\end{gathered}
$$

Let $a>0$ be a small positive constant. Changing $\sigma$ we can rewrite (5.28) as

$$
\left|\frac{\partial^{2} Y_{0}(t-\lambda, x-y ; \xi)}{\partial x_{i} \partial x_{j}}\right| \leq C(t-\lambda)^{-1+\frac{a \alpha}{2}}|x-y|^{-n-a} \exp \{-\sigma \rho(t, x ; \lambda, y)\} .
$$

Now we get by Lemma 3 and Lemma 4 that for $0<\varepsilon<\gamma$

$$
\begin{gathered}
I_{1}^{\prime} \leq C \int_{0}^{t}(t-\lambda)^{-1+\frac{a \alpha}{2}} \lambda^{-\alpha} d \lambda\left[\int_{\mathbb{R}^{n}}|x-y|^{-n+\gamma-\varepsilon-a}|x-\xi|^{-n+\varepsilon} \exp \{-\sigma \rho(t, x ; \lambda, y)\right. \\
\left.-\sigma \rho(\lambda, x ; 0, \xi)\} d y+\int_{\mathbb{R}^{n}}|x-y|^{-n+\gamma-\varepsilon-a}|y-\xi|^{-n+\varepsilon} \exp \{-\sigma \rho(t, x ; \lambda, y)-\sigma \rho(\lambda, y ; 0, \xi)\} d y\right] \\
=C|x-\xi|^{-n+\varepsilon} \exp \{-\sigma \rho(t, x ; 0, \xi)\} \int_{0}^{t}(t-\lambda)^{-1+\frac{a \alpha}{2}} \lambda^{-\alpha} d \lambda \\
\times \int_{\mathbb{R}^{n}}|x-y|^{-n+\gamma-\varepsilon-a} \exp \{-\sigma \rho(t, x ; \lambda, y)\} d y \\
+C \int_{0}^{t}(t-\lambda)^{-1+\frac{a \alpha}{2}} \lambda^{-\alpha} d \lambda \int_{\mathbb{R}^{n}}|x-y|^{-n+\gamma-\varepsilon-a}|y-\xi|^{-n+\varepsilon} \exp \{-\sigma \rho(t, x ; \lambda, y)-\sigma \rho(\lambda, y ; 0, \xi)\} d y \\
\leq C|x-\xi|^{-n+\varepsilon} \exp \{-\sigma \rho(\lambda, x ; 0, \xi)\} \int_{0}^{t}(t-\lambda)^{\frac{(\gamma-\varepsilon) \alpha}{2}-1} \lambda^{-\alpha} d \lambda \\
+C t^{-\alpha+\frac{a \alpha}{2}}|x-\xi|^{-n+\gamma-a} \exp \{-\sigma \rho(t, x ; 0, \xi)\}
\end{gathered}
$$

As before, the letters $\sigma, C$ meant various positive constants; this abuse of notation will be convenient in the sequel too. 
In order to obtain a bound for $I_{2}^{\prime}$, we use the estimate

$$
\begin{aligned}
& \left|\frac{\partial^{2} Y_{0}(t-\lambda, x-y ; y)}{\partial x_{i} \partial x_{j}}-\frac{\partial^{2} Y_{0}(t-\lambda, x-y ; \delta)}{\partial x_{i} \partial x_{j}}\right|_{\delta=x} \mid \\
\leq & C|x-y|^{\gamma-n}(t-\lambda)^{-1} \exp \{-\sigma \rho(t, x ; \lambda, y)\} \leq C|x-y|^{-n+\frac{\gamma}{2}}(t-\lambda)^{-1+\frac{\alpha \gamma}{4}} \exp \{-\sigma \rho(t, x ; \lambda, y)\}
\end{aligned}
$$

(with a different $\sigma$ ). Then by Lemma 4

$$
\begin{aligned}
& I_{2}^{\prime} \leq C|x-\xi|^{-n+\gamma} \exp \{-\sigma \rho(t, x ; 0, \xi)\} \\
& \quad \times \int_{0}^{t}(t-\lambda)^{-1+\frac{\alpha \gamma}{4}} \lambda^{-\alpha} d \lambda \int_{\mathbb{R}^{n}}|x-y|^{-n+\frac{\gamma}{2}} \exp \{-\sigma \rho(t, x ; \lambda, y)\} \\
& \leq C|x-\xi|^{-n+\gamma} \exp \{-\sigma \rho(t, x ; 0, \xi)\} \int_{0}^{t}(t-\lambda)^{-1+\frac{\alpha \gamma}{2}} \lambda^{-\alpha} d \lambda \\
& \leq C t^{\frac{\alpha \gamma}{2}-\alpha}|x-\xi|^{-n+\gamma} \exp \{-\sigma \rho(t, x ; 0, \xi)\} .
\end{aligned}
$$

Thus, we have proved (5.27) for $f(\lambda, y)=Q(\lambda, y ; \xi)$.

5.3. The fractional derivative. Our next task is to study the fractional derivative of the heat potential (5.22), again for the above two cases.

Suppose that $f(t, x)$ is bounded, locally Hölder continuous in $x$, and jointly continuous in $(t, x)$. We have $\mathbb{D}_{t}^{(\alpha)} W=\frac{\partial}{\partial t} v$ where $v=I_{0+}^{1-\alpha} W$ is the Riemann-Liouville fractional integral, that is

$$
\begin{aligned}
v(t, x)=\frac{1}{\Gamma(1-\alpha)} \int_{0}^{t}(t-\theta)^{-\alpha} d \theta \int_{0}^{\theta} d \lambda \int_{\mathbb{R}^{n}} Y_{0}(\theta-\lambda, x-y ; y) f(\lambda, y) d y \\
=\frac{1}{\Gamma(1-\alpha)} \int_{0}^{t} d \lambda \int_{\lambda}^{t}(t-\theta)^{-\alpha} d \theta \int_{\mathbb{R}^{n}} Y_{0}(\theta-\lambda, x-y ; y) f(\lambda, y) d y \\
=\int_{0}^{t} d \lambda \int_{\mathbb{R}^{n}} Z_{0}(t-\lambda, x-y ; y) f(\lambda, y) d y,
\end{aligned}
$$

by the definition of $Y_{0}$.

Let $h$ be a small positive number,

$$
v_{h}(t, x)=\int_{0}^{t-h} d \lambda \int_{\mathbb{R}^{n}} Z_{0}(t-\lambda, x-y ; y) f(\lambda, y) d y .
$$

Then $v_{h} \rightarrow v$ pointwise as $h \rightarrow 0$,

$$
\frac{\partial v_{h}}{\partial t}=v_{h}^{(1)}(t, x)+v_{h}^{(2)}(t, x)
$$


where

$$
\begin{gathered}
v_{h}^{(1)}(t, x)=\int_{\mathbb{R}^{n}} Z_{0}(h, x-y ; y) f(t-h, y) d y, \\
v_{h}^{(2)}(t, x)=\int_{0}^{t-h} d \lambda \int_{\mathbb{R}^{n}} \frac{\partial Z_{0}(t-\lambda, x-y ; y)}{\partial t} f(\lambda, y) d y .
\end{gathered}
$$

We have

$$
\begin{aligned}
v_{h}^{(1)}(t, x)=\int_{\mathbb{R}^{n}}\left[Z_{0}(h, x-y ; y)-Z_{0}(h, x-y ; x)\right] f(t-h, y) d y \\
\quad+\int_{\mathbb{R}^{n}} Z_{0}(h, x-y ; x)[f(t-h, y)-f(t-h, x)] d y+f(t-h, x),
\end{aligned}
$$

due to (4.13). By Proposition 3, the absolute value of the first integral in the right-hand side of (5.29) does not exceed

$$
C h^{-\alpha} \int_{\mathbb{R}^{n}}|x-y|^{-n+2+\gamma} \exp \left\{-\sigma\left(h^{-\alpha / 2}|x-y|\right)^{\frac{2}{2-\alpha}}\right\} d y=\text { const } \cdot h^{\frac{\gamma \alpha}{2}} \rightarrow 0,
$$

as $h \rightarrow 0$. Similarly, using (4.1) we show that the second integral in (5.29) tends to zero as $h \rightarrow 0$. Thus

$$
v_{h}^{(1)}(t, x) \longrightarrow f(t, x)
$$

as $h \rightarrow 0$.

Turning to $v_{h}^{(2)}$ we write

$$
\begin{aligned}
v_{h}^{(2)}(t, x)= & \int_{0}^{t-h} d \lambda \int_{\mathbb{R}^{n}} \frac{\partial Z_{0}(t-\lambda, x-y ; y)}{\partial t}[f(\lambda, y)-f(\lambda, x)] d y \\
& +\int_{0}^{t-h} f(\lambda, x) d \lambda \int_{\mathbb{R}^{n}} \frac{\partial Z_{0}(t-\lambda, x-y ; y)}{\partial t} d y .
\end{aligned}
$$

It follows from (4.15) that

$$
\left|\int_{0}^{t-h} d \lambda \int_{\mathbb{R}^{n}} \frac{\partial Z_{0}(t-\lambda, x-y ; y)}{\partial t}[f(\lambda, y)-f(\lambda, x)] d y\right| \leq C(t-\lambda)^{-1+\frac{\alpha \gamma}{2}} .
$$

Together with (4.16) this shows that

$$
\begin{aligned}
v_{h}^{(2)}(t, x) \longrightarrow \int_{0}^{t} d \lambda \int_{\mathbb{R}^{n}} \frac{\partial Z_{0}(t-\lambda, x-y ; y)}{\partial t}[f(\lambda, y)-f(\lambda, x)] d y \\
+\int_{0}^{t} f(\lambda, x) d \lambda \int_{\mathbb{R}^{n}} \frac{\partial Z_{0}(t-\lambda, x-y ; y)}{\partial t} d y .
\end{aligned}
$$


By the definition of $Y_{0}, \frac{\partial Z_{0}}{\partial t}=\mathbb{D}_{t}^{(\alpha)} Y_{0}$, and it follows from (5.30) and (5.31) that the fractional derivative $\mathbb{D}_{t}^{(\alpha)} W$ exists and can be represented as follows:

$$
\begin{aligned}
\left(\mathbb{D}_{t}^{(\alpha)} W\right)(t, x)=f(t, x)+\int_{0}^{t} d \lambda \int_{\mathbb{R}^{n}} \frac{\partial Z_{0}(t-\lambda, x-y ; y)}{\partial t}[f(\lambda, y)-f(\lambda, x)] d y \\
+\int_{0}^{t} f(\lambda, x) d \lambda \int_{\mathbb{R}^{n}} \frac{\partial Z_{0}(t-\lambda, x-y ; y)}{\partial t} d y .
\end{aligned}
$$

The case, in which $f(\lambda, y)=Q(\lambda, y ; \xi)$, is considered similarly, on the basis of the estimates $\left(4.15^{\prime}\right),(4.16),(5.17),(5.18)$.

5.4. The initial condition. The above study of heat potentials, together with the investigation of the integral $\int_{\mathbb{R}^{n}} Z_{0}(t, x-\xi) u_{0}(\xi) d \xi$ performed in [13 shows that our construction of the kernels $Z, Y$ indeed gives, via the formula (1.4), a solution of the equation (1.1). It remains to verify the initial condition (1.3).

By our construction,

$$
Z(t, x ; \xi)=Z_{0}(t, x-\xi, \xi)+V_{Z}(t, x ; \xi)
$$

where

$$
V_{Z}(t, x ; \xi)=\int_{0}^{t} d \lambda \int_{\mathbb{R}^{n}} Y_{0}(t-\lambda, x-y ; y) Q(\lambda, y ; \xi) d y
$$

(see (5.1)). We have

$$
\begin{aligned}
\int_{\mathbb{R}^{n}} Z_{0}(t, x-\xi, \xi) u_{0}(\xi) d \xi=\int_{\mathbb{R}^{n}} Z_{0}(t, x-\xi, x) & u_{0}(\xi) d \xi \\
& +\int_{\mathbb{R}^{n}}\left[Z_{0}(t, x-\xi, \xi)-Z_{0}(t, x-\xi, x)\right] u_{0}(\xi) d \xi .
\end{aligned}
$$

The first summand tends to $u_{0}(x)$, as $t \rightarrow 0$ [13. By Proposition 3, the second summand is majorized by

$$
\begin{aligned}
C t^{-\alpha} \int_{\mathbb{R}^{n}}|x-\xi|^{-n+2+\gamma} \exp \left\{-\sigma\left(t^{-\alpha / 2}|x-\xi|\right)^{\frac{2}{2-\alpha}}\right\} d \xi & \\
& =C t^{\gamma \alpha} \int_{\mathbb{R}^{n}}|\eta|^{-n+2+\gamma} \exp \left\{-\sigma|\eta|^{\frac{2}{2-\alpha}}\right\} d \eta \longrightarrow 0,
\end{aligned}
$$

as $t \rightarrow 0$. 
Let us find an estimate for $V_{Z}$. Suppose, for example, that $n=3$ (other cases are treated similarly). Then by (4.7) and (5.17)

$$
\left|V_{Z}(t, x ; \xi)\right| \leq C \int_{0}^{t}(t-\lambda)^{-\frac{\alpha}{2}-1} \lambda^{-\alpha} d \lambda \int_{\mathbb{R}^{3}}|y-\xi|^{-3+\gamma} \exp \{-\sigma[\rho(t, x ; \lambda, y)+\rho(\lambda, y ; 0, \xi)]\} d y .
$$

Taking $0<\gamma_{0}<\gamma$ we can rewrite this as

$$
\begin{aligned}
& \left|V_{Z}(t, x ; \xi)\right| \leq C \int_{0}^{t}(t-\lambda)^{-\frac{\gamma_{0} \alpha}{2}-1} \lambda^{-\alpha} d \lambda \int_{\mathbb{R}^{3}}\left(\frac{|x-y|}{(t-\lambda)^{\alpha / 2}}\right)^{1+\gamma_{0}}|x-y|^{-1-\gamma_{0}}|y-\xi|^{-3+\gamma} \\
& \times \exp \{-\sigma[\rho(t, x ; \lambda, y)+\rho(\lambda, y ; 0, \xi)]\} d y \\
& \leq C_{1} \int_{0}^{t}(t-\lambda)^{-\frac{\gamma_{0} \alpha}{2}-1} \lambda^{-\alpha} d \lambda \int_{\mathbb{R}^{3}}|x-y|^{-1-\gamma_{0}}|y-\xi|^{-3+\gamma} \exp \left\{-\sigma_{1}[\rho(t, x ; \lambda, y)+\rho(\lambda, y ; 0, \xi)]\right\} d y,
\end{aligned}
$$

and now Lemma 3 implies the estimate (iii) from the main Theorem.

Other estimates given in the formulation of our main Theorem are proved in a quite similar way (note that we roughen some estimates containing logarithmic terms replacing the logarithm by the power function with an arbitrarily small exponent). Returning now to the verification of the initial condition we obtain that

$$
\begin{aligned}
\left|\int_{\mathbb{R}^{3}} V_{Z}(t, x ; \xi) u_{0}(\xi) d \xi\right| \leq C t^{\frac{\gamma_{0} \alpha}{2}-\alpha} \int_{\mathbb{R}^{3}}|\xi|^{\gamma-\gamma_{0}-1} \exp \left\{-\sigma\left(t^{-\alpha / 2}|\xi|\right)^{\frac{2}{2-\alpha}}\right\} d \xi \\
=C t^{\frac{\gamma \alpha}{2}} \int_{\mathbb{R}^{3}}|\eta|^{\gamma-\gamma_{0}-1} \exp \left\{-\sigma|\eta|^{\frac{2}{2-\alpha}}\right\} d \eta \longrightarrow 0,
\end{aligned}
$$

as $t \rightarrow 0$. Thus, the initial condition (1.3) has been verified.

5.5. The Hölder continuity in $t$. Let us prove the assertion b) of the main theorem. Suppose that $0<\nu<1-\alpha, \alpha+\lambda<\nu$. We have to show that for any fixed $x \in \mathbb{R}^{n} t^{\nu} u(t, x)$ is Hölder continuous in $t$ with the exponent $\alpha+\lambda$. It is sufficient to prove the Hölder continuity near the origin $t=0$. Let us consider, for example, the case $n=3$; all other cases are treated similarly. 
Let us write $u(t, x)=u_{1}(t, x)+u_{2}(t, x)+u_{3}(t, x)+u_{4}(t, x)$ where

$$
\begin{gathered}
u_{1}(t, x)=\int_{\mathbb{R}^{3}} Z_{0}(t, x-\xi ; \xi) u_{0}(\xi) d \xi, \\
u_{2}(t, x)=\int_{\mathbb{R}^{3}} V_{Z}(t, x ; \xi) u_{0}(\xi) d \xi, \\
u_{3}(t, x)=\int_{0}^{t} d \lambda \int_{\mathbb{R}^{3}} Y_{0}(t-\lambda, x-\xi ; \xi) f(\lambda, \xi) d \xi, \\
u_{4}(t, x)=\int_{0}^{t} d \lambda \int_{\mathbb{R}^{3}} V_{Y}(t-\lambda, x ; \xi) f(\lambda, \xi) d \xi .
\end{gathered}
$$

The Hölder continuity of $t^{\nu} u_{1}(t, x)$ was proved in 13 (with the use of fractional calculus and the asymptotics of H-functions) for the case of constant coefficients. The proof for our case, in which there is also a dependence on the parameter $\xi$, is identical to [13].

As we saw above, $\left|u_{2}(t, x)\right| \leq C t^{\alpha \gamma / 2}$, so that $\left|t^{\nu} u_{2}(t, x)\right| \leq C t^{\frac{\alpha \gamma}{2}+\nu}<C t^{\alpha+\lambda}$ for small values of $t$, since $\alpha+\lambda<\nu$.

Next, by (4.7)

$\left|u_{3}(t, x)\right| \leq C \int_{0}^{t}(t-\lambda)^{-\frac{\alpha}{2}-1} d \lambda \int_{\mathbb{R}^{3}} \exp \left\{-\sigma\left(\frac{|x-\xi|}{(t-\lambda)^{\alpha / 2}}\right)^{\frac{2}{2-\alpha}}\right\} d \xi \leq C_{1} \int_{0}^{t}(t-\lambda)^{\alpha-1} d \lambda=C_{2} t^{\alpha}$,

so that $\left|t^{\nu} u_{3}(t, x)\right| \leq C_{2} t^{\alpha+\nu}<C_{2} t^{\alpha+\lambda}$ for small values of $t$.

Finally, from the estimate (iii) of the main Theorem we find that

$$
\left|t^{\nu} u_{4}(t, x)\right| \leq C t^{\frac{\alpha \gamma}{2}+\alpha+\nu}<C t^{\alpha+\lambda}
$$

for small values of $t$, as desired.

\section{THE ONE-DIMENSIONAL CASE}

In the case $n=1$ the parametrix and the fundamental solution have no singularity in the spatial variable. This simplifies the situation greatly and makes it similar to the theory of conventional parabolic equations of an arbitrary order. Note however that even this case is more complicated than the classical study of a second order parabolic differential equation. In particular, the estimates of iterated kernels in the Levi method should still be performed in two stages (see Lemma 6).

Here we give the main estimates for this case; their proofs, simplified versions of those given the preceding section, are omitted. The scheme of the Levi method and the main notations are as above; see (5.1)-(5.4). 
Proposition 7. If $n=1$, then

$$
\begin{gathered}
|M(t, x ; \xi)| \leq C t^{-(3-\gamma) \alpha / 2} \exp \{-\sigma \rho(t, x ; 0, \xi)\}, \\
|K(t, x ; \xi)| \leq C t^{-1-(1-\gamma) \alpha / 2} \exp \{-\sigma \rho(t, x ; 0, \xi)\}, \\
|Q(t, x ; \xi)| \leq C t^{-(3-\gamma) \alpha / 2} \exp \{-\sigma \rho(t, x ; 0, \xi)\}, \\
|\Psi(t, x ; \xi)| \leq C t^{-1-(1-\gamma) \alpha / 2} \exp \{-\sigma \rho(t, x ; 0, \xi)\} .
\end{gathered}
$$

The estimates for differences of the above kernels are collected in the next proposition.

Proposition 8. If $n=1$, then

$$
\begin{gathered}
\left|\Delta_{x} M(t, x ; \xi)\right| \leq C\left|x-x^{\prime}\right|^{\gamma-\varepsilon} t^{-(3-\varepsilon) \alpha / 2} \exp \left\{-\sigma \rho\left(t, x^{\prime \prime} ; 0, \xi\right)\right\}, \\
\left|\Delta_{x} K(t, x ; \xi)\right| \leq C\left|x-x^{\prime}\right|^{\gamma-\varepsilon} t^{-1-(1-\varepsilon) \alpha / 2} \exp \left\{-\sigma \rho\left(t, x^{\prime \prime} ; 0, \xi\right)\right\}, \\
\left|\Delta_{x} Q(t, x ; \xi)\right| \leq C\left|x-x^{\prime}\right|^{\gamma-\varepsilon} t^{-(3-\varepsilon) \alpha / 2} \exp \left\{-\sigma \rho\left(t, x^{\prime \prime} ; 0, \xi\right)\right\}, \\
\left|\Delta_{x} \Psi(t, x ; \xi)\right| \leq C\left|x-x^{\prime}\right|^{\gamma-\varepsilon} t^{-1-(1-\varepsilon) \alpha / 2} \exp \left\{-\sigma \rho\left(t, x^{\prime \prime} ; 0, \xi\right)\right\},
\end{gathered}
$$

$0<\varepsilon<\gamma$

\section{NONNEGATIVITY}

Let us prove that the functions $Z$ and $Y$ are nonnegative. It is sufficient to show the nonnegativity of the solution (1.4) of the problem (1.1), (1.3) for arbitrary nonnegative functions $u_{0} \in C_{0}^{\infty}\left(\mathbb{R}^{n}\right), f \in C_{0}^{\infty}\left([0, T] \times \mathbb{R}^{n}\right)$.

As we already know, the solution $u(t, x)$ is bounded (due to our estimates of $Z$ and $Y$ ), say $|u(t, x)| \leq M$, and belongs to $H_{\nu}^{\alpha+\lambda}[0, T], 0<\lambda<1-\alpha, \alpha+\lambda<\nu$, for each $x$. This regularity in $t$ makes it possible to represent the regularized fractional derivative $\mathbb{D}_{t}^{(\alpha)} u$ via the Marchaud fractional derivative:

$$
\left(\mathbb{D}_{t}^{(\alpha)} u\right)(t, x)=\frac{1}{\Gamma(1-\alpha)}\left[t^{-\alpha} u(t, x)-t^{-\alpha} u_{0}(x)\right]+\frac{\alpha}{\Gamma(1-\alpha)} \lim _{\varepsilon \rightarrow+0} \psi_{\varepsilon}(t, x)
$$

where $\psi_{\varepsilon}(t, x)=0$ for $0<t \leq \varepsilon$,

$$
\psi_{\varepsilon}(t, x)=\int_{0}^{t-\varepsilon} \frac{u(t, x)-u(\tau, x)}{(t-\tau)^{1+\alpha}} d \tau, \quad \varepsilon<t \leq T,
$$

and the limit exists for all $x \in \mathbb{R}^{n}, t \in[0, T]$ (see Sect. 13 in [21]).

Denote $v(t, x)=\frac{u(t, x)}{E_{\alpha}\left(\beta t^{\alpha}\right)}$ where $E_{\alpha}$ is the Mittag-Leffler function [9], $\beta>0$ will be chosen later. Using the fact that $\mathbb{D}_{t}^{(\alpha)} E_{\alpha}\left(\beta t^{\alpha}\right)=\beta E_{\alpha}\left(\beta t^{\alpha}\right)$ we obtain that

$$
\mathbb{D}_{t}^{(\alpha)} u(t, x)=\beta E_{\alpha}\left(\beta t^{\alpha}\right) v(t, x)-\frac{t^{-\alpha}}{\Gamma(1-\alpha)} u_{0}(x)+\left(L_{\beta} v\right)(t, x)
$$


where

$$
\left(L_{\beta} v\right)(t, x)=\frac{1}{\Gamma(1-\alpha)}\left[v(t, x) t^{-\alpha}+\alpha \lim _{\varepsilon \rightarrow+0} \int_{0}^{t-\varepsilon} E_{\alpha}\left(\beta \tau^{\alpha}\right) \frac{v(t, x)-v(\tau, x)}{(t-\tau)^{1+\alpha}} d \tau\right] .
$$

Now the equation (1.1) takes the form

$$
\frac{1}{E_{\alpha}\left(\beta t^{\alpha}\right)}\left(L_{\beta} v\right)(t, x)-(B-\beta) v(t, x)=g(t, x)
$$

where

$$
g(t, x)=\frac{1}{E_{\alpha}\left(\beta t^{\alpha}\right)}\left[\frac{t^{-\alpha}}{\Gamma(1-\alpha)} u_{0}(x)+f(t, x)\right] .
$$

Let $c_{0}=\sup _{x \in \mathbb{R}^{n}}|c(x)|, d>0, \beta=c_{0}+d$. Consider the function

$$
F_{R}(t, x)=\frac{M}{R^{2}}\left(|x|^{2}+\mu t^{\alpha}+1\right), \quad R, \mu>0 .
$$

Since $F_{R}(t, x)$ and $E_{\alpha}\left(\beta t^{\alpha}\right)$ are monotone increasing in $t$, we have

$$
\left(L_{\beta} F_{R}\right)(t, x) \geq\left(D_{0+}^{\alpha} F_{R}\right)(t, x) \geq \frac{M \mu}{R^{2}} D_{0+}^{\alpha}\left(t^{\alpha}\right)=\frac{M \mu \Gamma(1+\alpha)}{R^{2}} .
$$

Here $D_{0+}^{\alpha}$ is the Riemann-Liouville fractional derivative (see [21]), and in estimating $L_{\beta} F_{R}$ we used the Marchaud form of $D_{0+}^{\alpha}$.

On the other hand,

$$
\begin{aligned}
(B-\beta) F_{R}(t, x)=\sum_{i=1}^{n} a_{i i}(x) \frac{2 M}{R^{2}}+\sum_{j=1}^{n} b_{j}(x) \frac{2 M}{R^{2}} x_{j}+ & {\left[c(x)-c_{0}\right] F_{R}(t, x)-d F_{R}(t, x) } \\
\leq & \frac{M}{R^{2}}\left(C_{1}+C_{2}|x|-d|x|^{2}-d \mu t^{\alpha}-d\right),
\end{aligned}
$$

so that

$\frac{1}{E_{\alpha}\left(\beta t^{\alpha}\right)}\left(L_{\beta} F_{R}\right)(t, x)-(B-\beta) F_{R}(t, x) \geq \frac{M}{R^{2}}\left[\frac{\mu \Gamma(1+\alpha)}{E_{\alpha}\left(\beta t^{\alpha}\right)}-C_{1}-C_{2}|x|+d|x|^{2}+d \mu t^{\alpha}+d\right] \geq 0$

for all $x \in \mathbb{R}^{n}, t \in[0, T]$, if the number $\mu$ is large enough.

Let $G(t, x)=v(t, x)+F_{R}(t, x)$. Since $g(t, x) \geq 0$, it follows from (7.1) and (7.2) that

$$
\frac{1}{E_{\alpha}\left(\beta t^{\alpha}\right)}\left(L_{\beta} G\right)(t, x)-(B-\beta) G(t, x) \geq 0 .
$$

If $|x|=R$, then

$$
G(t, x)=v(t, x)+M+\frac{\mu M t^{\alpha}}{R^{2}}+\frac{M}{R^{2}}>0 .
$$

Also $G(0, x)=u_{0}(x)+F_{R}(0, x)>0$. Then $G(t, x) \geq 0$ for all $(t, x)$ with $t \in[0, T],|x|<R$. Indeed, otherwise the function $G$ possesses a point $\left(t^{0}, x^{0}\right)$ of its global minimum on the set 
$\{0<t \leq T,|x|<R\}$, such that $G\left(t^{0}, x^{0}\right)<0$. At this point $(B-\beta) G\left(t^{0}, x^{0}\right) \geq 0$ [10, and by (7.3) $\left(L_{\beta} G\right)\left(t^{0}, x^{0}\right) \geq 0$, which contradicts the definition of $L_{\beta}$.

Thus, if $|x| \leq R$, then

$$
u(t, x) \geq-\frac{M}{R^{2}}\left(|x|^{2}+\mu t^{\alpha}+1\right) E_{\alpha}\left(\beta t^{\alpha}\right) .
$$

Since $R$ is arbitrary, this means that $u(t, x) \geq 0$.

\section{References}

[1] V. V. Anh and N. N. Leonenko, Spectral analysis of fractional kinetic equations with random data, J. Statist. Phys. 104 (2001), 1349-1387.

[2] B. Baeumer and M. Meerschaert, Stochastic solutions for fractional Cauchy problems, Fract. Calc. Appl. Anal. 4 (2001), 481-500.

[3] E. Bazhlekova, The abstract Cauchy problem for fractional evolution equation, Fract. Calc. Appl. Anal. 1 (1998), 255-270.

[4] E. Bazhlekova, Fractional Evolution Equations in Banach Spaces, Dissertation, Technische Universiteit Eindhoven, 2001.

[5] B. L. J. Braaksma, Asymptotic expansions and analytic continuation for a class of Barnes integrals, Compositio Math. 15 (1964), 239-341.

[6] M. M. Dzhrbashyan and A. B. Nersessyan, Fractional derivatives and Cauchy problem for differential equations of fractional order, Izv. AN Arm. SSR. Matematika 3 (1968), 3-29 (Russian).

[7] S. D. Eidelman, Parabolic Systems, North-Holland, Amsterdam, 1969.

[8] A. M. El-Sayed, Fractional order evolution equations, J. Fract. Calc. 7 (1995), 89-100.

[9] A. Erdelyi, W. Magnus, F. Oberhettinger, and F. Tricomi, Higher Transcendental Functions. Vol. III, McGraw-Hill, New York, 1955.

[10] A. Friedman, Partial Differential Equations of Parabolic Type, Prentice-Hall, Englewood Cliffs, NJ, 1964.

[11] R. Gorenflo, F. Mainardi, D. Moretti, and P. Paradisi, Time fractional diffusion: A discrete random walk approach, Nonlinear Dynamics 29 (2002), 129-143.

[12] A. N. Kochubei, A Cauchy problem for evolution equations of fractional order, Differential Equations 25 (1989), 967-974.

[13] A. N. Kochubei, Fractional-order diffusion, Differential Equations 26 (1990), 485-492. 
[14] T. Kolsrud, On a class of probabilistic integrodifferential equations. In: Ideas and Methods in Mathematics and Physics. Memorial Volume Dedicated to Raphael Høegh-Krohn, Vol. 1, Cambridge University Press, 1992, pp. 168-172.

[15] V. A. Kostin, Cauchy problem for an abstract differential equation with fractional derivatives, Russian Acad. Sci. Dokl. Math. 46 (1993), 316-319.

[16] O. A. Ladyzhenskaya, V. A. Solonnikov, and N. N. Uraltseva, Linear and Quasilinear Equations of Parabolic Type, American Mathematical Society, Providence, 1968.

[17] M. M. Meerschaert, D. A. Benson, H. P. Scheffler and B. Baeumer, Stochastic solutions of space-time fractional diffusion equations, Phys. Rev. E 65 (2002), 1103-1106.

[18] R. Metzler and J. Klafter, The random walk's guide to anomalous diffusion: a fractional dynamics approach, Physics Reports, 339 (2000), 1-77.

[19] K. Miller and B. Ross, An Introduction to the Fractional Calculus and Fractional Differential Equations, Wiley and Sons, New York, 1993.

[20] A. P. Prudnikov, Yu. A. Brychkov, and O. I. Marichev, Integrals and Series. Vol. 3: More Special Functions, Gordon and Breach, New York, 1990.

[21] S. G. Samko, A. A. Kilbas, and O. I. Marichev, Fractional Integrals and Derivatives: Theory and Applications, Gordon and Breach, New York, 1993.

[22] W. R. Schneider, W. Wyss, Fractional diffusion and wave equations, J. Math. Phys. 30 (1989), 134-144.

[23] W. R. Schneider, Fractional diffusion, Lecture Notes Phys., 355 (1990), 276-286.

[24] W. R. Schneider, Grey noise. In: Ideas and Methods in Mathematics and Physics. Memorial Volume Dedicated to Raphael Høegh-Krohn, Vol. 1, Cambridge University Press, 1992, pp. 261-282.

[25] H. M. Srivastava, K. C. Gupta, and S. P. Goyal, The H-Functions of One and Two variables with Applications, South Asian Publishers, New Dehli, 1982.

[26] W. Wyss, The fractional diffusion equation, J. Math. Phys. 27 (1986), 2782-2785.

[27] M. Yor, W. Schneider's grey noise and fractional Brownian motion. In: Proc. Easter Meeting on Probability (Edinburgh, April 10-14, 1989). 